IFIC/05-29

FTUV/05-0704

UG-FT-190-05

CAFPE-60-05

\title{
Interpolating between low and high energy QCD via a 5D Yang-Mills model
}

\author{
Johannes Hirn ${ }^{a}$ and Verónica Sanz ${ }^{b}$ \\ ${ }^{a}$ IFIC, Departament de Física Teòrica, CSIC - Universitat de València \\ Edifici d'Instituts de Paterna, Apt. Correus 22085, 46071 València, Spain \\ e-mail: johannes.hirn@ific.uv.es \\ ${ }^{b}$ Departamento de Física Teórica y del Cosmos, Universidad de Granada \\ Campus de Fuentenueva, 18071 Granada, Spain \\ e-mail: vsanz@ugr.es
}

\begin{abstract}
We describe the Goldstone bosons of massless QCD together with an infinite number of spin-1 mesons. The field content of the model is $\mathrm{SU}\left(N_{f}\right) \times \mathrm{SU}\left(N_{f}\right)$ Yang-Mills in a compact extra-dimension. Electroweak interactions reside on one brane. Breaking of chiral symmetry occurs due to the boundary conditions on the other brane, away from our world, and is therefore spontaneous. Our implementation of the holographic recipe maintains chiral symmetry explicit throughout. For intermediate energies, we extract resonance couplings. These satisfy sum rules due to the 5D nature of the model. These sum rules imply, when taking the high-energy limit, that perturbative QCD constraints are satisfied. We also illustrate how the 5D model implies a definite prescription for handling infinite sums over $4 \mathrm{D}$ resonances. Taking the low-energy limit, we recover the chiral expansion and the corresponding non-local order parameters. All local order parameters are introduced separately.
\end{abstract}




\section{Contents}

1. Introduction 2

2. The setup 1

2.1 The symmetries and the symmetry-breaking pattern

2.1.1 $5 \mathrm{D} \mathrm{SU}\left(N_{f}\right) \times \mathrm{SU}\left(N_{f}\right)$ gauge invariance

2.1.2 Symmetry breaking by BCs

2.1.3 The spectrum 6

2.1.4 Conformal invariance in the UV

3. Chiral implementation of the holographic recipe 7

3.1 The generating functional 8

3.1.1 Green's functions of QCD currents

3.1.2 From $5 \mathrm{D}$ to $4 \mathrm{D}$

3.2 The $A_{5}$ and the pion field

3.2.1 GBs as the Wilson line 9

3.2.2 Redefinitions for vector fields 10

3.2 .3 Derived $\mathrm{BCs}$

4. Very low energies: Goldstone bosons 11

$4.1 \chi \mathrm{PT}$ lagrangian at $\mathcal{O}\left(p^{2}\right)$

4.2 Predictions for $\chi \mathrm{PT}$ lagrangian at $\mathcal{O}\left(p^{4}\right)$

5. Intermediate energies: spin-1 resonances 14

5.1 Resonances as Kaluza-Klein excitations 15

5.2 Couplings of resonances 16

6. High energies: sums of resonance contributions 17

6.1 Sums over resonance couplings 17

6.2 Vector form factor 18

$6.2 .14 \mathrm{D}$ point of view 18

$6.2 .25 \mathrm{D}$ point of view 19

6.3 Froissart bound 20

6.4 Axial form factor 21

6.5 Lightest vector-meson dominance 21

7. High energies: two-point functions and OPE 22

7.1 The vector and axial two-point functions 22

7.1.1 Explicit expression for RS1

7.2 The left-right correlator 25

$7.34 \mathrm{D}$ point of view 26

7.4 Condensates 27 
8. Conclusions 27

A. Field redefinitions 29

B. Resonance wave-functions 31

G. Resonance couplings 33

D. 5D regularization of infinite sums 34

D.1 KK regularization 35

D.2 Generalized Weinberg sum rules 36

\section{Introduction}

We model spin-1 resonances and the massless pions of QCD in the chiral limit. The aim is to improve the understanding of the interplay between low-energy hadron physics and the known behavior of perturbative QCD. For this purpose, we build and explore a model of $\mathrm{SU}\left(N_{f}\right) \times \mathrm{SU}\left(N_{f}\right)$ Yang-Mills $(\mathrm{YM})$ fields with a compact extra dimension. We stress that the fifth dimension is used as a tool here, and is not meant to be physical. From the fourdimensional (4D) point of view, the spectrum contains an infinite number of resonances, as expected in large- $N_{c}$ QCD [1, 2]. Note that we limit ourselves to $\mathrm{SU}\left(N_{f}\right)$ rather than $\mathrm{U}\left(N_{f}\right)$ for simplicity.

To discuss a model of mesons, it is appropriate to first take into account the symmetry constraints on the lightest mesons: the pions, which are the Goldstone bosons (GBs) of the spontaneously broken $4 \mathrm{D} \mathrm{SU}\left(N_{f}\right) \times \mathrm{SU}\left(N_{f}\right)$ chiral symmetry. These symmetry constraints are embodied by Chiral Perturbation Theory $(\chi \mathrm{PT})$ [3, 4, 5]. To go beyond the range of validity of this effective theory, i.e. to energies higher than the mass of the $\rho$ resonance, one resorts to models of resonances: various models exist for the $\rho$ and $a_{1}$ [6, 0, 8, 9]. Besides pions, these resonances play the leading role in low-energy hadron physics [10]. Their couplings are constrained by requiring matching with the high-energy theory [11].

In order to recover perturbative QCD logarithms in two-point functions, one needs a model with either an infinite number of resonances or a perturbative continuum. Many efforts have been devoted recently to explore the constraints imposed on these models 12, 13, 14, 15, 16, 17, 18] by the matching with both $\chi \mathrm{PT}$ at low energies and QCD at high energies. Namely, this tells us about the symmetries, the soft high-energy behavior and the bearing of QCD at large- $N_{c}$ on the model.

A five-dimensional (5D) model naturally describes such an infinite number of states: with a compact extra dimension, each $5 \mathrm{D}$ field can alternatively be seen as a tower of Kaluza-Klein (KK) excitations. We are interested in such an infinite tower of massive spin-1 fields, supposedly describing the vector and axial resonances $\left(\rho, a_{1}, \rho^{\prime}, \cdots\right)$ [19, 20]. Having a lagrangian formalism allows us to go beyond two-point functions. In addition, the 
a priori infinite number of resonance parameters are fixed by only one gauge coupling and the geometry, described by one additional parameter. These two parameters correspond to $N_{c}$ and $\Lambda_{\mathrm{QCD}}$.

The fact that the spin-1 mesons come from the same 5D YM field has deeper implications than just labeling the resonances. In the 5D model, higher-dimensional gauge invariance is realized among the meson interactions not only as infinitely many (higgsed) 4D gauge invariances, but it also contains a residual symmetry [21], whose GBs describe the pions. Since the pions descend from the original YM field, their interactions with mesons are constrained. As we show in this paper, this ensures certain relations constraining the high-energy behavior of various amplitudes.

To understand the origin of this soft high-energy behavior, it is useful to go back to those 4D models which introduced the $\rho$ meson as a YM field [6, 7]. As shown in [11], such models automatically exhibit a soft high-energy behavior for some amplitudes, as required by QCD. The models could be generalized to include the $a_{1}$ meson [8]. Using gauge fields at the sites of a latticized/deconstructed extra-dimension [22], one can even describe an infinite tower of vector and axial mesons [19]. However, there is a fundamental ingredient added in the 5D gauge theory: exact locality in the extra dimension. This implies an even softer high-energy behavior [23].

An independent motivation to consider a 5D model with YM fields comes from the AdS/CFT correspondence [24, 25, 26, 27]. This correspondence established an equivalence between two very precise theories, one defined in $4 \mathrm{D}$, and the other in $5 \mathrm{D}$. None of them has any known phenomenological applications. The bet here is to use a simple idea behind the AdS/CFT: a global symmetry in $4 D$ corresponds to its gauged version realized in the $5 D$ bulk, and to see how a 5D YM model can mimic QCD.

Notice that the soft high-energy behavior given by the 5D YM model may be welcome from the point of view of QCD. This is the case for the vector form factor, as we show in this paper. When undesirable, the too-soft high-energy behavior should be corrected in order to agree with QCD. For instance, 5D locality implies an infinite set of vanishing sum rules [28, 29], generalizing those known as Weinberg sum rules (WSRs) in QCD [30, 31, 32]. This high-energy behavior is even softer than in QCD, in which the presence of a quarkantiquark condensate leaves us with only two vanishing Weinberg sum rules (in the chiral limit). This discordance can be cured by the adjunction of scalar degrees of freedom in the bulk (corresponding to the quark condensate and its spin-0 excitations) [23, 33, 34]. We avoid this complication here: while still retaining many features of QCD and a good agreement with phenomenology, this allows us to push analytical computations further. We also propose a way of introducing the $\langle\bar{q} q\rangle$ condensate without additional degrees of freedom.

The simplicity of our model comes from the way the spontaneous breaking of the 4 D global $\mathrm{SU}\left(N_{f}\right) \times \mathrm{SU}\left(N_{f}\right)$ symmetry is implemented: by identifying gauge couplings away from our world, i.e. at a distance in the fifth dimension. This breaking can be communicated to our world only by infrared effects, and will only generate non-local order parameters of chiral symmetry. The virtue is that the 5D YM fields yield a multiplet of pions coming from the fifth component of the axial vector field $A_{5}$, without the need for 
any field other than 5D YM.

This is different from the 5D model of [33, 34], which takes into account a quark condensate, and in which bulk pseudo-scalars mix with the $A_{5}$. The inclusion of quark condensate, quark masses and spin-0 resonances in our model is deferred to future work. Even without these ingredients, we have the usual set of non-local order parameters, with non-vanishing values given by the low energy constants of the chiral lagrangian: the $L_{i}$ 's, and first and foremost, $f_{\pi}^{2}$. In our model, all local order parameters, including $\langle\bar{q} q\rangle$, are treated on an equal footing: they must be introduced separately in the model as infrared (IR) modifications of the metric ${ }^{1}$. We present a general method to introduce condensates, whether order parameters or not.

From the $4 \mathrm{D}$ point of view, our treatment is also different. After describing the setup in Section 2, we introduce in Section 3 a specific implementation of the holographic recipe that separates explicitly between sources and fields. In Section 1 , we pay particular attention to the chiral symmetries and to the matching with very low energies; i.e. $\chi$ PT.

Regarding intermediate energies (Section 5), we obtain the resonance spectra and couplings. These parameters satisfy sum rules: in Section 6 we show that this results in a soft high-energy behavior, as expected in QCD. Two-point functions of quark currents are considered in Section 7: the leading logarithm of perturbative QCD is recovered. We show how to reproduce the condensates in the OPE without introducing additional degrees of freedom. We also explain in what sense the model gives a prescription for handling infinite sums over resonances in Appendix D.

\section{The setup}

The model will contain the essential features in order to interpolate between the known behavior at low and high energies of Green's functions of the QCD currents. It is directly formulated in terms of mesons, and never refers to quarks. Our inputs are the following: (i) a five-dimensional space-time and (ii) $5 \mathrm{D} \mathrm{SU}\left(N_{f}\right) \times \mathrm{SU}\left(N_{f}\right)$ gauge symmetry, broken by boundary conditions.

The outputs will be: (i) spontaneously broken $4 \mathrm{D} \mathrm{SU}\left(N_{f}\right) \times \mathrm{SU}\left(N_{f}\right)$ symmetry and its GBs, the pions, (ii) an infinite number of vector and axial resonances with a clear prescription for handling them and, (iii) soft behavior at high energies.

If one further asks for conformal invariance in part of the geometry, partonic logarithms can be recovered.

We write the $5 \mathrm{D}$ metric as

$$
\mathrm{d} s^{2}=g_{M N} \mathrm{~d} x^{M} \mathrm{~d} x^{N}, \quad \text { with } M, N=0,1,2,3,5,
$$

where we will denote the coordinates as $\left(x^{\mu}, z\right)$ with $\mu=0, \cdots, 3$ and $x^{5} \equiv z$ representing the position in the fifth dimension. The signature is $(+----)$. We will keep general

\footnotetext{
${ }^{1}$ Note that, as in the model of [33, 34], an independent parameter has to be introduced for the quark condensate. This has no equivalent in QCD.
} 
covariance in most of the computations, but always assume that the metric can be put in the conformally flat form

$$
\mathrm{d} s^{2}=w(z)^{2}\left(\eta_{\mu \nu} \mathrm{d} x^{\mu} \mathrm{d} x^{\nu}-\mathrm{d} z^{2}\right) .
$$

Such a metric enjoys 4D Poincaré invariance for each hypersurface of constant $z$. The extra dimension considered here is an interval ${ }^{2}$. The two ends of the space are located at $l_{0}$ (the $U V$ brane) and $l_{1}$ (the $I R$ brane), with the names UV/IR implying $w\left(l_{0}\right) \geqslant w\left(l_{1}\right)$ for the warp factor of (2.2).

\subsection{The symmetries and the symmetry-breaking pattern}

We wish to study Green's functions of the quark currents of QCD. For this, we construct a model of mesons. The lagrangian, as well as the expressions for the currents will involve only bosons.

\subsubsection{D SU$\left(N_{f}\right) \times \mathrm{SU}\left(N_{f}\right)$ gauge invariance}

The global 4D chiral symmetry is upgraded to gauge symmetry in the bulk, whose particular realization at one special point $z=l_{0}$ (UV brane) has to be identified with the chiral symmetry of QCD. As already mentioned in the Introduction, this 5D gauged version of the chiral symmetry finds its origin in the AdS/CFT correspondence. This fixes the field content: we consider a $5 \mathrm{D} \mathrm{SU}\left(N_{f}\right) \times \mathrm{SU}\left(N_{f}\right)$ gauge theory.

The model includes in one step an infinite number of resonances: they are the 4D excitations of the same 5D YM field. Regarding the lagrangian, we limit ourselves in this paper to operators involving no more than two derivatives, and work at tree level ${ }^{3}$. Therefore, the description is completely fixed once one specifies the $5 \mathrm{D}$ gauge coupling and the metric: the action is

$$
\mathcal{S}_{\mathrm{YM}}=-\frac{1}{4 g_{5}^{2}} \int \mathrm{d}^{4} x \int_{l_{0}}^{l_{1}} \mathrm{~d} z \sqrt{g} g^{M N} g^{R S}\left\langle L_{M R} L_{N S}+R_{M R} R_{N S}\right\rangle,
$$

where $\langle\cdots\rangle$ means the trace in flavor space, and $R_{M N} \equiv \partial_{M} R_{N}-\partial_{N} R_{M}-\mathrm{i}\left[R_{M}, R_{N}\right]$. The square of the $5 \mathrm{D}$ YM coupling $g_{5}^{2}$ has dimensions of length. The action (2.3) is invariant under "parity" $L \leftrightarrow R$ and the $5 \mathrm{D} \mathrm{SU}\left(N_{f}\right) \times \mathrm{SU}\left(N_{f}\right)$ gauge transformations denoted by $R(x, z), L(x, z)$ acting as $R_{M} \equiv R_{M}^{a} \frac{T^{a}}{\sqrt{2}} \longmapsto R R_{M} R^{\dagger}+\mathrm{i} R \partial_{M} R^{\dagger}$, with the generators of $\mathrm{SU}\left(N_{f}\right)$ normalized by $\left\langle T^{a} T^{b}\right\rangle=2 \delta^{a b}$.

The 5D gauge fields $L_{M}$ and $R_{M}$ enjoy a higher-dimensional gauge invariance. From the $4 \mathrm{D}$ point of view, $5 \mathrm{D}$ gauge invariance is seen as an infinite number of $4 \mathrm{D}$ gauge invariances plus a shift symmetry [21, 37]. In Section 3.2 we will make use of this residual $4 \mathrm{D}$ symmetry to identify the pions. In QCD, the pions are the GBs of chiral symmetry breaking. Here, the pions will be the GBs of the shift symmetry, the remnant of the 5D gauge symmetry.

\footnotetext{
${ }^{2}$ The interval is more suitable than the orbifold for our purposes 35. Indeed, in Section B, we will need to consider more general boundary conditions than the ones used in the orbifold approach: the values of the fields at one boundary will play the role of classical sources for the generating functional.

${ }^{3}$ The hope is that a consistent treatment at the level of loops can be formulated as an effective theory. The action would then have to include operators with more derivatives: derivative and loop expansion should be related, using power-counting rules such as those in the Appendices of [36].
} 


\subsubsection{Symmetry breaking by BCs}

We break the symmetry by boundary conditions, which distinguish between the vector and axial combinations of the $L_{M}$ and $R_{M}$ fields. This will produce the spontaneous breaking of chiral symmetry at low energies. We impose at the IR brane

$$
R_{\mu}\left(x, z=l_{1}\right)-L_{\mu}\left(x, z=l_{1}\right)=0 .
$$

This enforces the equality of the $5 \mathrm{D}$ gauge transformations $R$ and $L$ at the point $z=l_{1}$. We call $h(x)$ their common value

$$
h(x) \equiv R\left(x, z=l_{1}\right) \quad=\quad L\left(x, z=l_{1}\right) .
$$

For the vector combination, we adopt a gauge-invariant condition

$$
R_{5 \mu}\left(x, z=l_{1}\right)+L_{5 \mu}\left(x, z=l_{1}\right)=0 .
$$

This simple breaking is enough to give the correct order of magnitude for the $\chi \mathrm{PT}$ lowenergy constants, $L_{i}$ 's. Essentially, the way it works from the $4 \mathrm{D}$ point of view is by lifting the axial tower of resonances from the vector ones. This will produce at low energies a non-zero decay constant of the pion, $f$.

\subsubsection{The spectrum}

Given the field content and symmetry breaking pattern just described, the mass eigenstates will be the axial and vector combinations of the $L_{M}$ and $R_{M}$ gauge fields, $A_{M}$ and $V_{M}$. Two remarks are in order here: the Lorentz index $M$ takes 5 values, i.e. $A_{M}=\left(A_{\mu}, A_{5}\right)$ and each component $A_{M}\left(x^{\mu}, z\right)$ is a linear combination of an infinite number of $4 \mathrm{D}$ fields, $A_{M}^{(n)}(x)$, weighted by a profile in the fifth dimension, $\varphi_{n}^{A}(z)$. This is the so-called KK-decomposition

$$
A_{M}(x, z)=\sum_{n=0}^{\infty} \varphi_{n}^{A}(z) A_{M}^{(n)}(x),
$$

where the values of the profiles are completely determined by the geometry and the BCs.

Given our BCs, the physical spectrum will be composed by (i) a multiplet of massless $4 \mathrm{D}$ particle, related to the zero mode of the axial field, the $A_{5}^{(0)}(x)$ and (ii) two sets of $4 \mathrm{D}$ massive vector fields, $A_{\mu}^{(n)}$ and $V_{\mu}^{(n)}$, where $n$ runs from 1 to $\infty$. The other KK modes

are either absent due to the BCs $\left(\right.$ no $\left.V_{\mu}^{(0)}, A_{\mu}^{(0)}, V_{5}^{(0)}\right)$, or eaten via higgsing: the $V_{5}^{(n)}$ and $A_{5}^{(n)}$ (with $n \neq 0$ ) feed the longitudinal components of the $4 \mathrm{D}$ vector fields $V_{\mu}^{(n)}, A_{\mu}^{(n)}$.

After imposing the BCs (2.4, 2.6), part of the full 5D gauge invariance is broken. The residual symmetries are: (i) chiral symmetry (or shift symmetry) protecting the GBs and (ii) higgsed $\prod_{n}\left[\mathrm{SU}\left(N_{f}\right) \times \mathrm{SU}\left(N_{f}\right)\right]_{(n)}$ gauge invariance enjoyed by $A_{\mu}^{(n)}$ and $V_{\mu}^{(n)}$.

\subsubsection{Conformal invariance in the UV}

Consider the two-point correlators $\Pi_{V, A}$ of quark currents $J_{V, A}^{a \mu}=\bar{q} \gamma^{\mu}\left(\gamma_{5}\right) \frac{T^{a}}{2} q$

$$
2 \mathrm{i} \int \mathrm{d}^{4} x \mathrm{e}^{\mathrm{i} q \cdot x}\left\langle 0\left|T\left\{J_{V, A}^{a \mu}(x) J_{V, A}^{b \nu}(0)\right\}\right| 0\right\rangle=\delta^{a b}\left(q^{\mu} q^{\nu}-q^{2} \eta^{\mu \nu}\right) \Pi_{V, A}\left(q^{2}\right) .
$$


In large- $N_{c}$ QCD with vanishing quark masses, the OPE expansion of $\Pi_{V, A}$ gives, for large euclidean momentum $Q^{2} \equiv-q^{2}[31]$

$$
\Pi_{V, A}\left(-Q^{2}\right)=-\frac{N_{c}}{12 \pi^{2}}\left\{\lambda+\log \left(\frac{Q^{2}}{\mu^{2}}\right)+\mathcal{O}\left(\alpha_{s}\right)\right\}+\alpha_{s} \kappa_{4} \frac{\langle G G\rangle}{Q^{4}}+\alpha_{s} \kappa_{6}^{V, A} \frac{\langle\bar{q} q\rangle^{2}}{Q^{6}}+. .
$$

where dimensional regularization has introduced a term $\lambda=\mu^{d-4} \frac{2}{d-4}+\gamma_{E}-\ln (4 \pi)+$ $\ln \left(\mu^{2}\right)+\mathcal{O}(d-4)$. The first term in (2.9) is the dimension-0 operator obtained from perturbative $\mathrm{QCD}$, the second is a gluon condensate, and the third involves the quark condensate. For the 5D model to reproduce the partonic logarithm, the metric has to be conformally/scale invariant near the UV brane: although the result is known in $5 \mathrm{D}$, we will reobtain it explicitly in the computation of the two-point function, equation (7.10).

For most of this paper, we adopt the simplest geometry that reproduces the dimension0 term in the OPE of $\Pi_{V, A}$. We consider a space-time warped by the Anti-de-Sitter (AdS) curvature

$$
w(z)=\frac{l_{0}}{z} .
$$

The metric is then exactly conformal, and conformal invariance is broken only by the presence of the IR brane, as in the model of Randall and Sundrum [38], RS1.

With the warp factor $(2.10)$ and our field content, one cannot reproduce the remaining terms in the OPE of $\Pi_{V, A}(2.9)$. To remedy this, deviations from conformality should be introduced near the IR brane: in Section 7.4 we simply sketch the consequences for the case of the two-point correlators (2.8). With this in mind, we derive results for a generic metric, except in explicit computations. We also illustrate the dependence on the metric through numerical comparison between RS1 and the flat space (FS).

\section{Chiral implementation of the holographic recipe}

Quarks and gluons provide the correct description of QCD at high energies whereas, at low energies, $\chi \mathrm{PT}$ is the procedure to compare with experiment. We intend to link both sides of QCD via a model of GBs and spin-1 mesons, without aiming for the full generality of an effective theory.

Resemblance with QCD will come from various aspects. The straightforward reason why a 5D YM model could be of any help to interpolate between QCD and $\chi \mathrm{PT}$ is the immediate inclusion of an infinite number of resonances which respect the correct symmetries (chiral and scale invariance). Other reasons to look at a 5D model are hints: holography and the AdS/CFT correspondence, in addition to the partial successes of hidden local symmetry approaches.

In this Section we introduce the so-called holographic recipe derived from the AdS/CFT correspondence, used to construct the Green's functions of currents. This is a 5D-specific implementation of the external field method. The external field method is also often used in descriptions of QCD in terms of mesons 伯. It introduces sources to generate the Noether currents of the $4 \mathrm{D} \mathrm{SU}\left(N_{f}\right) \times \mathrm{SU}\left(N_{f}\right)$ global symmetry, see Section 3.1. We show how to go from the $5 \mathrm{D}$ version to the $4 \mathrm{D}$ formalism: in Section 3.2, we define pions and vector fields that transform covariantly with respect to the chiral symmetry. 


\subsection{The generating functional}

\subsubsection{Green's functions of QCD currents}

The generating functional of the QCD Noether currents $J_{L, R}^{\mu}=\bar{q} \gamma^{\mu} \frac{1 \mp \gamma_{5}}{2} \frac{T^{a}}{2} q$ of the global $\mathrm{SU}\left(N_{f}\right) \times \mathrm{SU}\left(N_{f}\right)$ symmetry is defined through the functional integral over gluons $G$ and quarks $q$

$$
\mathcal{Z}_{\mathrm{QCD}}\left[\ell_{\mu}, r_{\mu}\right]=\mathrm{e}^{\mathrm{i} \Gamma_{\mathrm{QCD}}\left[\ell_{\mu}, r_{\mu}\right]}=\int[\mathrm{d} G][\mathrm{d} q] \mathrm{e}^{\mathrm{i} \int\left(\mathcal{L}_{\mathrm{QCD}}+\ell_{\mu}^{a} J_{L}^{a \mu}+r_{\mu}^{a} J_{R}^{a \mu}\right)} .
$$

In the above, $\ell_{\mu}(x)=\ell_{\mu}^{a} \frac{T^{a}}{2}$ and $r_{\mu}(x)=r_{\mu}^{a} \frac{T^{a}}{2}$ are classical configurations coupled to the QCD currents.

Green's functions obtained from $\Gamma_{\mathrm{QCD}}$ satisfy all Ward identities of the global $4 \mathrm{D}$ $\mathrm{SU}\left(N_{f}\right) \times \mathrm{SU}\left(N_{f}\right)$ symmetry. The whole set of Ward identities is equivalent to the fact that $\Gamma_{\mathrm{QCD}}\left[\ell_{\mu}, r_{\mu}\right]$ is invariant under a local version of the $4 \mathrm{D} \mathrm{SU}\left(N_{f}\right) \times \mathrm{SU}\left(N_{f}\right)$ symmetry [4, 39, 40] which acts on the sources $\ell_{\mu}, r_{\mu}$ as

$$
\begin{aligned}
\ell_{\mu} \longmapsto g_{L} \ell_{\mu} g_{L}^{\dagger}+\mathrm{i} g_{L} \partial_{\mu} g_{L}^{\dagger}, \\
r_{\mu} \longmapsto g_{R} r_{\mu} g_{R}^{\dagger}+\mathrm{i} g_{R} \partial_{\mu} g_{R}^{\dagger},
\end{aligned}
$$

i.e. the sources are gauge connections for the local version of chiral symmetry. In addition, if one coupled QCD to the electroweak interactions, a subset of these sources would become the dynamical photon and $W^{ \pm}, Z^{0}$ fields: this yields the right interactions of QCD currents with the electroweak vector bosons.

Consider now another generating functional describing the same Green's functions in terms of physical fields at low energies, e.g. pions for $\chi \mathrm{PT}$. In order for its Green's functions to obey the same Ward identities, one requires invariance of the action under the local chiral symmetry, acting on the mesons and the sources. We now turn to our model, and show that it does indeed satisfy this requirement.

\subsubsection{From 5D to $4 \mathrm{D}$}

We start with the 5D action (2.3), integrated over the 5D YM gauge fields

$$
\mathcal{Z}_{\text {model }}=\int\left[\mathrm{d} L_{M}\right]\left[\mathrm{d} R_{M}\right] \mathrm{e}^{\mathrm{i} \mathcal{S}_{\mathrm{YM}}\left[L_{M}, R_{M}\right]} .
$$

The holographic recipe in addition defines that the 5D YM fields should satisfy the following constraint: their value at the UV boundary is a classical configuration

$$
\begin{aligned}
& L_{\mu}\left(x, z=l_{0}\right)=\ell_{\mu}(x), \\
& R_{\mu}\left(x, z=l_{0}\right)=r_{\mu}(x),
\end{aligned}
$$

on which the generating functional depends. This holographic recipe will allow us to extract the Noether currents of the global $\mathrm{SU}\left(N_{f}\right) \times \mathrm{SU}\left(N_{f}\right)$ symmetry, expressed in terms of mesons.

The generating functional is then invariant under the local transformations of the sources (3.2 3.3): this $4 \mathrm{D}$ local invariance is the $5 \mathrm{D}$ gauge invariance at a specific point 
$z=l_{0}$. Therefore, we see hat the Green's functions will satisfy the QCD Ward identities. Note that electroweak interactions in this model reside on the UV brane, where the sources are. Integrating over the boundary values $\ell_{\mu}, r_{\mu}$ would amount to considering dynamical $W^{ \pm}, Z^{0}$ and photon.

After redefining the fields so as to explicitly respect chiral symmetry in Section 3.2 and extracting the pions, we will perform the KK decomposition. This will leave us with two infinite towers of massive vector fields $\left(V_{\mu}^{(n)}, A_{\mu}^{(n)}\right)$, the classical sources $\left(\ell_{\mu}, r_{\mu}\right)$ and the massless pion modes, collected in the unitary matrix $U$. We will be able to rewrite the generating functional as

$$
\mathcal{Z}_{\text {model }}\left[\ell_{\mu}, r_{\mu}\right]=\int[\mathrm{d} U]\left[\mathrm{d} V_{\mu}^{(n)}\right]\left[\mathrm{d} A_{\mu}^{(n)}\right] \mathrm{e}^{\mathrm{i} \int d^{4} x\left(\mathcal{L}_{2}^{\chi \mathrm{PT}}+\mathcal{L}_{4}^{\chi \mathrm{PT}}+\mathcal{L}_{\text {resonances }}\right)}
$$

where $\left(\mathcal{L}_{2}^{\chi \mathrm{PT}}+\mathcal{L}_{4}^{\chi \mathrm{PT}}\right)\left[U, \ell_{\mu}, r_{\mu}\right]$ are the $\mathcal{O}\left(p^{2}\right)$ and $\mathcal{O}\left(p^{4}\right)$ chiral lagrangians. All terms involving resonances are collected in $\mathcal{L}_{\text {resonances }}\left[U, V_{\mu}^{(n)}, A_{\mu}^{(n)}, \ell_{\mu}, r_{\mu}\right]$ : kinetic and mass terms, as well as interactions with pions and sources. At energies of the order of the resonance masses, the action in (3.7) is the appropriate one, since it describes the interactions of each resonance separately. We will obtain and describe it in Section 5. At energies lower than the KK scale $p^{2} \ll \pi^{2} / l_{1}^{2}$, one can integrate out the massive states: the low energy action for GBs $\mathcal{L}_{2}^{\chi \mathrm{PT}}+\mathcal{L}_{4}^{\chi \mathrm{PT}}$ is then obtained, with definite predictions for the low-energy constants (Section 4). At the other extreme, i.e. high energies, it is more convenient to use the 5D description, see Section 0.

\subsection{The $A_{5}$ and the pion field}

We mentioned the spectrum of our model in Section 3.1.2. In particular, contrary to the 5D model by [33, 34], we have introduced only YM fields: the spectrum contains a massless 4D spin-0 mode, due to the chosen boundary conditions. It is related to the (axial combination of) the fifth component of the gauge fields. In this section, we show how to extract the GB field with appropriate symmetry properties. Details are relegated to Appendix A.

\subsubsection{GBs as the Wilson line}

We first define Wilson lines $\xi$ extending from $l_{1}$ to $z$ along the fifth coordinate $x^{5}$, but for a fixed point $x^{\mu}$ in $4 \mathrm{D}$ space

$$
\xi_{R}(x, z) \equiv \mathrm{P}\left\{\mathrm{e}^{\mathrm{i} \int_{l_{1}}^{z} \mathrm{~d} x^{5} R_{5}\left(x, x^{5}\right)}\right\},
$$

with $\mathrm{P}$ the path-order integral. Using one such Wilson line to go from the UV brane to the IR brane with the $L_{5}$ field, and back with the $R_{5}$ field, we define the $4 \mathrm{D}$ object $U$

$$
U(x) \equiv \xi_{R}\left(x, l_{0}\right) \xi_{L}^{\dagger}\left(x, l_{0}\right) .
$$

Given the properties of the Wilson line, $U$ obeys the following transformation law under the $5 \mathrm{D}$ gauge transformations

$$
U(x) \longmapsto g_{R}(x) U(x) g_{L}^{\dagger}(x) .
$$


Equation (3.10) involves only the elements $\left(g_{R}, g_{L}\right)$ of the $4 \mathrm{D} \mathrm{SU}\left(N_{f}\right) \times \mathrm{SU}\left(N_{f}\right)$ symmetry. It is the appropriate transformation law for GBs of the breaking to the vector $\mathrm{SU}\left(N_{f}\right)$ 41. With a vacuum such that $R_{5}=0$ and $L_{5}=0$ up to a gauge, we see that, in our model, the $4 \mathrm{D}$ symmetry is spontaneously broken to its vector subgroup. Indeed, the vacuum is $U=1$, which is invariant only if $g_{R}=g_{L}$.

\subsubsection{Redefinitions for vector fields}

We have extracted the pion field as a product of Wilson lines extending from one boundary to the other. We also recall the property of the Wilson line $\xi$ from $l_{1}$ to a point in the bulk at position $z$

$$
\xi_{R}^{\dagger}\left(\partial_{5}-\mathrm{i} R_{5}\right) \xi_{R}=0
$$

This shows that the fifth component of the gauge fields could be gauged away by using $\xi$ as the gauge transformation. However, such a gauge transformation depending on the value of fields is more appropriately treated as a field redefinition, rather than a gauge fixing [42, 43]. One then defines, the following vector and axial combinations of gauge fields

$$
\hat{V}_{M}, \hat{A}_{M} \equiv \mathrm{i}\left\{\xi_{L}^{\dagger}\left(\partial_{M}-\mathrm{i} L_{M}\right) \xi_{L} \pm(L \rightarrow R)\right\} .
$$

Note that their fifth component vanish, $\hat{V}_{5}=0, \hat{A}_{5}=0$.

The next step is to separate the hatted quantities into dynamical fields and external sources. This is done by subtracting from the hatted quantities (3.12), their values at the boundary, times the appropriate profile depending on $z$. The UV boundary value of $\hat{V}_{\mu}$ is still related to the sources, so this subtraction indeed separates sources and fields. For the vector case, subtracting a constant profile in $z$ is adequate

$$
V_{\mu}(x, z) \equiv \hat{V}_{\mu}(x, z)-\hat{V}_{\mu}\left(x, l_{0}\right) .
$$

As for the axial combination, its UV boundary value involves the derivative of the pion field in addition to the axial combination of the sources

$$
\left.\hat{A}_{\mu}(x, z)\right|_{z=l_{0}}=-\frac{\mathrm{i}}{2} \xi_{R}^{\dagger}\left(x, l_{0}\right)\left(D_{\mu} U\right) \xi_{L}\left(x, l_{0}\right) .
$$

In that case, we need to introduce a function $\alpha(z)$ such that

$$
A_{\mu}(x, z) \equiv \hat{A}_{\mu}(x, z)-\alpha(z) \hat{A}_{\mu}\left(x, l_{0}\right) .
$$

The appropriate expression for $\alpha(z)$ will be provided later in Section 1 . It will follow uniquely from the requirement of diagonalizing quadratic terms in the action, and therefore turns out to depend only on the metric.

We have thus constructed fields that transform homogeneously under the adjoint representation of $h(x)$

$$
\begin{aligned}
& V_{\mu}(x, z) \longmapsto h(x) V_{\mu}(x, z) h(x)^{\dagger}, \\
& A_{\mu}(x, z) \longmapsto h(x) A_{\mu}(x, z) h(x)^{\dagger} .
\end{aligned}
$$


This implementation of the holographic recipe is very close to the external field method traditionally used in $\chi \mathrm{PT}$ [4]. Another advantage of our procedure is that we avoid gaugefixing. This is of paramount importance in order to track down the chiral symmetry, which severely constrains the interactions of the GBs.

\subsubsection{Derived BCs}

We now derive the BCs that the new fields $V_{\mu}, A_{\mu}$ satisfy. This is done by injecting the above definitions for the fields $V_{\mu}, A_{\mu}$ into the BCs for the original fields $R_{M}, L_{M}$ on the IR brane (2.4) and (2.6). We get ${ }^{4}$

$$
\begin{aligned}
\left.V_{\mu}\right|_{l_{0}} & =0, \\
\left.\partial_{5} V_{\mu}\right|_{l_{1}} & =0 .
\end{aligned}
$$

This means that the dynamical field $V_{\mu}$ satisfies what we will call $(-,+)$ BCs. The UV $\mathrm{BC}$ directly follows from having performed the subtraction (3.13).

Regarding the axial field $A_{\mu}$, we first have to specify BCs for the function $\alpha(z)$, which enters into the definition (3.15). We choose the following, which will yield simple BCs for $A_{\mu}$

$$
\begin{aligned}
& \alpha\left(l_{0}\right)=1, \\
& \alpha\left(l_{1}\right)=0 .
\end{aligned}
$$

Once these $\mathrm{BCs}$ for the function $\alpha$ are chosen, it follows that $A_{\mu}$ satisfies $(-,-)$ BCs, i.e.

$$
\begin{aligned}
& \left.A_{\mu}\right|_{l_{0}}=0, \\
& \left.A_{\mu}\right|_{l_{1}}=0 .
\end{aligned}
$$

Having appropriately subtracted boundary values from the covariant combinations of $L_{\mu}, R_{\mu}$, we have obtained $V_{\mu}, A_{\mu}$ fields with simple BCs. The sources have been separated, and will appear explicitly in the lagrangian, as opposed to implicitly via EOMs in (3.4).

\section{Very low energies: Goldstone bosons}

In this paper, we propose a model of mesons which interpolates between QCD at low and high energies. We first focus on low energies, where symmetries are the main handle: the properties of the GBs follow from the (broken) symmetry. Since the model implements the appropriate spontaneous breaking, we will recover the non-linear GBs derivative interactions, provided our way of treating the model respects the symmetries. This was the reason behind the non-standard non-linear treatment of Section 3 .

The $\chi \mathrm{PT}$ lagrangian is expanded according to powers of four-dimensional derivatives. Simply counting the number of $4 \mathrm{D}$ Lorentz indices in the two terms of the 5D YM action

\footnotetext{
${ }^{4}$ This is a covariant expression, i.e. $\partial_{5} V_{\mu} \longmapsto h\left(\partial_{5} V_{\mu}\right) h^{\dagger}$.
} 
$F_{\mu 5}^{2}$ and $F_{\mu \nu}^{2}$, one can see where the $\mathcal{O}\left(p^{2}\right)$ and $\mathcal{O}\left(p^{4}\right)$ terms of the $\chi \mathrm{PT}$ lagrangian come from in our model: we write schematically

$$
\begin{aligned}
& \left\langle F_{5 \mu}\right\rangle^{2} \rightarrow \mathcal{L}_{2}^{\chi \mathrm{PT}}+\text { resonance mass terms, } \\
& \left\langle F_{\mu \nu}\right\rangle^{2} \rightarrow \mathcal{L}_{4}^{\chi \mathrm{PT}}+\text { resonance kinetic \& interaction terms. }
\end{aligned}
$$

Since this is a model with two parameters, we will obtain predictions for the $\chi \mathrm{PT}$ low-energy constants. The explicit results are derived in this Section and Appendix A. Covariance with respect to $4 \mathrm{D}$ chiral symmetry is automatic, due to the work done in Section 3, so the identification with the $\chi \mathrm{PT}$ lagrangian holds strictly.

As is usual in models where resonances are introduced as fields with one (4D) Lorentz index as opposed to tensor fields, the contribution of resonance exchange to GB interactions for $p^{2} \ll M_{\rho}^{2}$ starts at $\mathcal{O}\left(p^{6}\right)$ [11. Thanks to the work of the previous Section, "integrating out" resonances is trivial at $\mathcal{O}\left(p^{2}\right)$ and $\mathcal{O}\left(p^{4}\right)$ : one simply reads off the GB interactions from the lagrangian $\mathcal{L}_{2}^{\chi \mathrm{PT}}+\mathcal{L}_{4}^{\chi \mathrm{PT}}$, disregarding operators involving resonance fields.

\section{1 $\chi$ PT lagrangian at $\mathcal{O}\left(p^{2}\right)$}

The rewriting (4.1) is directly obtained from

$$
2\left\langle L_{5 \mu} L_{5 \nu}+R_{5 \mu} R_{5 \nu}\right\rangle=\left(\partial_{5} \alpha\right)^{2}\left\langle D_{\mu} U D_{\nu} U^{\dagger}\right\rangle+4\left\langle\partial_{5} V_{\mu} \partial_{5} V_{\nu}+\partial_{5} A_{\mu} \partial_{5} A_{\nu}\right\rangle .
$$

The first term in the right-hand side will yield a kinetic term for the GBs, once integrated over the fifth coordinate. The second term will yield masses for the KK modes of $V_{\mu}$ and $A_{\mu}$, after integration by parts.

In fact, there should be an additional term in (4.3), producing a mixing between the pion and the axial resonances. However, if we ask that $\alpha$ satisfies the following wave equation

$$
\partial_{5}\left(\sqrt{g} g^{\mu \nu} g^{55} \partial_{5}\right) \alpha=0
$$

then, the mixing vanishes. We indeed have the freedom to require (4.4), since the function $\alpha$ was up to now completely undetermined, except for its BCs (3.203.21). Note that (4.4) is a massless spin-1 equation. This was expected, since $\alpha(z)$ is the wave-function of $D_{\mu} U(x)$, i.e. of the derivative of a massless spin-0 field. Bear in mind however, that $\alpha$ has to satisfy unusual BCs (3.20 3.21).

Our rewritings yield the standard GB interactions involving two derivatives

$$
\begin{aligned}
& -\frac{2}{4 g_{5}^{2}} \int_{l_{0}}^{l_{1}} \mathrm{~d} z \sqrt{g} g^{55} g^{\mu \nu}\left\langle R_{5 \mu} R_{5 \nu}+L_{5 \mu} L_{5 \nu}\right\rangle \\
= & \frac{f^{2}}{4}\left\langle D_{\mu} U D^{\mu} U^{\dagger}\right\rangle+\frac{1}{2} \sum_{n}\left\langle M_{V_{n}}^{2} V_{\mu}^{(n)} V^{(n) \mu}+M_{A_{n}}^{2} A_{\mu}^{(n)} A^{(n) \mu}\right\rangle,
\end{aligned}
$$

with an expression for the pion decay constant $f$

$$
\eta^{\mu \nu} f^{2} \equiv-\frac{1}{g_{5}^{2}} \int_{l_{0}}^{l_{1}} \mathrm{~d} z \sqrt{g} g^{55} g^{\mu \nu}\left(\partial_{5} \alpha\right)^{2} .
$$


The first term in the right-hand side of $(4.5)$ is the chiral-invariant $\mathcal{O}\left(p^{2}\right)$ kinetic and interaction term of GBs, including their coupling to sources: it is strictly that of [5]. One also obtains a second term in the right-hand side of (4.5), to be interpreted later in Section 5.1 as the sum of the mass terms for the KK modes.

Solving for $\alpha$ (Appendix A), the general expression for $f(4.6)$ yields

$$
\left.f^{2}\right|_{\mathrm{FS}, \mathrm{RS} 1}=\frac{1}{\left(g_{4} l_{1}\right)^{2}}, \frac{2}{\left(g_{4} l_{1}\right)^{2}}
$$

for the flat space and RS1 metrics respectively. In this result, the limit $l_{0} \longrightarrow 0$ has already been taken. For RS1, this limit can be safely taken only for physical quantities, provided in addition $l_{0} / g_{5}^{2}$ remains finite. Indeed, one can define the $4 \mathrm{D}$ gauge coupling

$$
\left.\frac{1}{g_{4}^{2}}\right|_{\mathrm{FS}, \mathrm{RS} 1}=\frac{l_{1}}{g_{5}^{2}}, \frac{l_{0}}{g_{5}^{2}},
$$

which should be kept finite ${ }^{5}$.

\subsection{Predictions for $\chi \mathbf{P T}$ lagrangian at $\mathcal{O}\left(p^{4}\right)$}

Particularizing to $N_{f}=3$, we turn our attention to the next-to-leading order interactions in the chiral expansion. Such GB $\mathcal{O}\left(p^{4}\right)$ interactions are produced by those terms in the $5 \mathrm{D}$ Yang-Mills lagrangian that contain four $4 \mathrm{D}$ Lorentz indices, i.e. $\left\langle R_{\mu \nu} R_{\rho \sigma}+L_{\mu \nu} L_{\rho \sigma}\right\rangle$, as described in (4.2). We provide the full rewritings in Appendix A: this directly yields interaction terms of GBs and sources $r_{\mu}, \ell_{\mu}$ respecting chiral symmetry, in the form of the $\mathcal{O}\left(p^{4}\right)$ lagrangian of [5]. One then simply identifies the free low-energy constants describing the interactions of GBs at next-to-leading order. Their values are given by integrals of polynomials of the function $\alpha$ over the fifth coordinate. We find explicitly

$$
\begin{aligned}
\eta^{\mu \nu} \eta^{\rho \sigma} L_{1} & =\frac{1}{32 g_{5}^{2}} \int_{l_{0}}^{l_{1}} \mathrm{~d} z \sqrt{g} g^{\mu \rho} g^{\nu \sigma}\left(1-\alpha^{2}\right)^{2}, \\
\eta^{\mu \nu} \eta^{\rho \sigma} L_{10} & =-\frac{1}{4 g_{5}^{2}} \int_{l_{0}}^{l_{1}} \mathrm{~d} z \sqrt{g} g^{\mu \rho} g^{\nu \sigma}\left(1-\alpha^{2}\right), \\
\eta^{\mu \nu} \eta^{\rho \sigma} H_{1} & =-\frac{1}{8 g_{5}^{2}} \int_{l_{0}}^{l_{1}} \mathrm{~d} z \sqrt{g} g^{\mu \rho} g^{\nu \sigma}\left(1+\alpha^{2}\right) .
\end{aligned}
$$

The other $L_{i}$ constants are given in terms of the previous ones by

$$
\begin{aligned}
L_{2} & =2 L_{1}, \\
L_{3} & =-6 L_{1}, \\
L_{9}+L_{10} & =0 .
\end{aligned}
$$

These relations hold for any metric, and deserve some comments. First of all, since we have introduced neither scalar sources nor quark masses, the $\mathcal{O}\left(p^{4}\right)$ constants $L_{4}, L_{5}, L_{6}, L_{7}, H_{2}$ do not appear.

\footnotetext{
${ }^{5} g_{4}$ would correspond to the $4 \mathrm{D}$ YM coupling after dimensional reduction.
} 
Second, we find the relation $L_{2}=2 L_{1}$, as expected in large- $N_{c}$ 《5. We also find $L_{3}=-6 L_{1}$, which is standard for models with one multiplet of vector resonances. It comes directly from the Skyrme structure of the $\mathcal{O}\left(p^{4}\right)$ GB interaction term, which we find here as was the case in the extra-dimensional model of [20] and hidden local symmetry models [7]. Consequences on GB forward scattering amplitudes will be discussed in Section 6.3 .

Third, we find the relation $L_{9}+L_{10}=0$. This is more unusual, and will have consequences on the axial form factor in Section 6.4. In 4D models with only the $\rho$ resonance (no higher resonances, and no axial resonances), it was also obtained as a consequence of the KSFR I relation [11]. (The result was however modified by the adjunction of the $a_{1}$.) In our model, it holds in the presence of both towers of vector and axial resonances: the two come from linear combinations of the same 5D YM fields.

In Table 1, we give the analytic expressions for the $L_{i}$ 's: we consider two examples for the metric: flat space $w(z)=1$ and RS1 $w(z)=l_{0} / z$ where the limit $l_{0} \longrightarrow 0$ with $g_{4}$ (4.8) finite is assumed.

As in any resonance model used at tree-level, the predictions for the $L_{i}$ 's are scale-independent. Indeed, in the limit of large number of colors it is expected that the leading order consists of tree diagrams involving mesons [10] ${ }^{6}$. Presumably, the predictions for the $L_{i}$ 's should be interpreted as the values of the renormalized constants at some scale $\mu$ of the order of the mass of the first resonances. A

\begin{tabular}{|c|c|c|}
\hline & $(z)=1$ & $w(z)=\frac{l_{0}}{z}$ \\
\hline$f^{2}$ & $\frac{1}{\left(g_{4} l_{1}\right)^{2}}$ & $\frac{2}{\left(g_{4} l_{1}\right)^{2}}$ \\
\hline$L_{1}$ & $\frac{1}{60 g_{4}^{2}}$ & $\frac{11}{768 g_{4}^{2}}$ \\
\hline$L_{2}$ & $2 L_{1}$ & $2 L_{1}$ \\
\hline$L_{3}$ & $-6 L_{1}$ & $-6 L_{1}$ \\
\hline$L_{9}$ & $\frac{1}{6 g_{4}^{2}}$ & $\frac{3}{16 g_{4}^{2}}$ \\
\hline$L_{10}$ & $-L_{9}$ & $-L_{9}$ \\
\hline
\end{tabular}

Table 1: Formulas for lowenergy constants. comparison of these predictions with the values extracted from $\mathcal{O}\left(p^{4}\right)$ fits [47], with the scale $\mu=M_{\rho}$ is presented for our two backgrounds in Table 2 .

To obtain numerical values, we have to input the two independent parameters of the model: $g_{4}$ and $l_{1}$. For this, we invert the exact formulas yielding $M_{V_{1}}$ and $f$, and adjust them to the experimentally measured $M_{\rho} \simeq 776 \mathrm{MeV}$ and $f_{\pi} \simeq 87 \mathrm{MeV}$ in the chiral limit.

\begin{tabular}{|c|c|c|c|}
\hline & $w(z)=1$ & $w(z)=\frac{l_{0}}{z}$ & Experiment \\
\hline $10^{3} L_{1}$ & 0.52 & 0.52 & $0.4 \pm 0.3$ \\
\hline $10^{3} L_{2}$ & 1.03 & 1.04 & $1.35 \pm 0.3$ \\
\hline $10^{3} L_{3}$ & -3.10 & -3.12 & $-3.5 \pm 1.1$ \\
\hline $10^{3} L_{9}$ & 5.2 & 6.8 & $6.9 \pm 0.7$ \\
\hline $10^{3} L_{10}$ & -5.2 & -6.8 & $-5.5 \pm 0.7$ \\
\hline
\end{tabular}
We stick to this simple procedure through- Table 2: Numerical values for the $L_{i}$ coefficients. out the paper since our model is one of large- $N_{c}$ QCD, and we therefore do not expect fine numerical agreement (in particular for resonance masses). As a matter of fact, the only reason why we keep three digits for $L_{1}, L_{2}, L_{3}$ is not because we trust them, but to show the difference between the two metrics.

\section{Intermediate energies: spin-1 resonances}

The KK modes of the 5D model we propose are to be interpreted as mesons: this is the description appropriate at intermediate energies. The link between QCD and this model of mesons treated at tree level is provided by the limit of a large number of colors. In

${ }^{6}$ Note that the formalism of antisymmetric tensor fields is currently being used for treating the lightest resonances at the level of quantum loops 44]. This may remedy the lack of scale dependence of the $L_{i}$ 's obtained by integration of the resonances at tree-level 445, 46]. 
this limit, QCD is equivalent to a theory involving an infinite number of infinitely narrow states. Our model treats an infinite tower of states of spin-1, in addition to the GBs. In this Section, properties and interactions of resonances are extracted.

\subsection{Resonances as Kaluza-Klein excitations}

Following the standard KK reduction procedure, we assume separation of variables, i.e. that a $5 \mathrm{D}$ vector field $V_{\mu}(x, z)$ satisfying the EOMs derived from the $5 \mathrm{D}$ lagrangian can be decomposed as a sum of $4 \mathrm{D}$ modes $V_{\mu}^{(n)}(x)$, each possessing a profile $\varphi_{n}^{V}(z)$ in the fifth dimension

$$
V_{\mu}(x, z)=\sum_{n} V_{\mu}^{(n)}(x) \varphi_{n}^{V}(z)
$$

From the EOMs, we find that the profiles $\varphi_{n}^{V}$ have to satisfy the following differential equation for a Sturm-Liouville operator

$$
\eta^{\mu \nu} \partial_{5}\left(\sqrt{g} g^{\rho \sigma} g^{55} \partial_{5}\right) \varphi_{n}^{V}=\sqrt{g} g^{\mu \nu} g^{\rho \sigma} M_{V_{n}}^{2} \varphi_{n}^{V}
$$

with $(-,+)$ BCs. $M_{V_{n}}^{2}>0$ will be identified as the mass of the $4 \mathrm{D}$ KK mode $V^{(n)}$. The same procedure can be applied to the $A_{\mu}(x, z)$ field, with $(-,-)$ BCs. Explicit formulas for wave-functions are collected in Appendix B for the case of flat space and RS1.

\begin{tabular}{|l|c|c|}
\hline & $w(z)=1$ & $w(z)=\frac{l_{0}}{z}$ \\
\hline$M_{V_{n}}$ & $\frac{\pi}{l_{1}}\left(n-\frac{1}{2}\right)$ & $\frac{\pi}{l_{1}}\left(n-\frac{1}{4}\right)$ \\
\hline$M_{A_{n}}$ & $\frac{\pi}{l_{1}} n$ & $\frac{\pi}{l_{1}}\left(n+\frac{1}{4}\right)$ \\
\hline
\end{tabular}

Table 3: Approximate expressions for the masses of heavy resonances.

Approximate expressions for the masses of the heavy resonances $M_{V_{n}, A_{n}}, n \gg 1$ are given in Table 3. The indexing is such that $\rho$ and $a_{1}$ are labeled by $n=1$, i.e. correspond to the KK modes $V^{(1)}$ and $A^{(1)}$ respectively. These expressions are approximate at a few percent level for $n \sim 1$ in the case of RS1, and exact for flat space. The exact value for the rho mass is equal to $\pi /\left(2 l_{1}\right)$ in flat space and $2.4 / l_{1}$ in RS1. For heavy excitations $n \gg 1$, the behavior for these $5 \mathrm{D}$ models is

$$
M_{V_{n}, A_{n}}^{2} \propto n^{2} .
$$

This behavior of the masses with $n$ is a consequence of the 5D Lorentz invariance broken by the finite size of the extra-dimension. This may seem unsatisfactory, given the known result for large- $N_{c}$ QCD in $1+1$ dimensions [48] and the Regge behavior [49]. Nonetheless, there exists no proof that $M_{n}^{2} \sim n$ is the right behavior for $n \gg 1$ in large- $N_{c}$ QCD in $4 \mathrm{D}$.

Another objection that may be put forward regards the spectrum of the light resonances. In RS1 with the limit $l_{0} \rightarrow 0$, the ratio $M_{A_{1}} / M_{V_{1}}$ is approximately 1.6 , in agreement with the experimental value of $M_{a_{1}} / M_{\rho}$. However, in our model, the quick growth of the interspacing between resonances as $n$ increases is already felt for the lightest modes, implying that the masses for $\rho^{\prime}, \rho^{\prime \prime}$ are higher than the experimental values quoted by [50], see Table 1 . In flat space, the problem would be worse. 
We note that this can in principle be cured directly by including modifications of the $\mathrm{AdS}_{5}$ metric near the IR brane. At any rate, the leading order in $1 / N_{c}$ falls short of taking into account the non-negligible

\begin{tabular}{|c|c|c|c|}
\hline & $w(z)=1$ & $w(z)=\frac{l_{0}}{z}$ & Experiment \\
\hline$M_{A_{1}}$ & $1.6 \mathrm{GeV}$ & $1.2 \mathrm{GeV}$ & $1230 \pm 40 \mathrm{MeV}$ \\
\hline$M_{V_{2}}$ & $2.3 \mathrm{GeV}$ & $1.8 \mathrm{GeV}$ & $1465 \pm 25 \mathrm{MeV}$ \\
\hline$M_{V_{3}}$ & $3.9 \mathrm{GeV}$ & $2.8 \mathrm{GeV}$ & $1688.1 \pm 2.1 \mathrm{MeV}$ \\
\hline
\end{tabular}

Table 4: Numerical values for lightest resonance masses. width of QCD resonances.

\subsection{Couplings of resonances}

The original 5D YM lagrangian contains interactions between 5D fields. These entail, via the KK decomposition (5.1), definite resonance interactions. In Appendix C, we give expressions for couplings involving one resonance as bulk integrals of polynomials in $\alpha$ times the appropriate factors of the wave-functions.

Using the notations of [51], generalized to resonances indexed by $n$, the expressions of Table 5 show the behavior of the couplings $f_{V_{n}}, f_{A_{n}}, g_{V_{n}}$ for large $n^{7}$. Once again, these expressions are approximate in the case of RS1, and exact for flat space. For RS1, the approximation is again good up to a few percents, even for $n=1$, except for $g_{V_{n}}$, for which the few-percent level accuracy is reached only for $n>15$. Indeed, even the sign of $g_{V_{1}}$ would

\begin{tabular}{|c|c|c|}
\hline & $w(z)=1$ & $w(z)=\frac{l_{0}}{z}$ \\
\hline$f_{V_{n}}$ & $\frac{\sqrt{2}}{\pi g_{4}} \frac{1}{n-\frac{1}{2}}$ & $\frac{1}{g_{4}} \frac{1}{\sqrt{n-\frac{1}{4}}}$ \\
\hline$f_{A_{n}}$ & $\frac{\sqrt{2}}{\pi g_{4}} \frac{1}{n}$ & $\frac{1}{g_{4}} \frac{1}{\sqrt{n+\frac{1}{4}}}$ \\
\hline$g_{V_{n}}$ & $\frac{\sqrt{2}}{\pi^{3} g_{4}} \frac{1}{\left(n-\frac{1}{2}\right)^{3}}$ & $\frac{(-1)^{n}}{8 \sqrt{2} \pi^{2} g_{4}} \frac{1}{\left(n-\frac{1}{4}\right)^{2}}$ \\
\hline
\end{tabular}

Table 5: Approximate formulas for resonance parameters. be wrong if one used the asymptotic formula.

Table 6 collects the results for $\rho$ decays. Inputs are $f=87 \mathrm{MeV}$ and $M_{\rho}=776$ $\mathrm{MeV}$. The first column corresponds to a model with only one vector resonance: the $\rho$, after imposing the KSFR I \& II relations $g_{\rho}^{2} M_{\rho}^{2}=f^{2} / 2$ and $f_{\rho}=2 g_{\rho}$ to fix $g_{\rho}$ and $f_{\rho}$. The 5D RS1 model is certainly not disfavored compared to the KSFR predictions in the chiral limit.

\begin{tabular}{|c|c|c|c|c|}
\hline & KSFR & $w(z)=1$ & $w(z)=\frac{l_{0}}{z}$ & Experiment \\
\hline$\Gamma\left(\rho \rightarrow e^{+} e^{-}\right) \mathrm{KeV}$ & 4.4 & 4.4 & 8.1 & $7.02 \pm 0.11$ \\
\hline$\Gamma(\rho \rightarrow \pi \pi) \mathrm{MeV}$ & 205 & 135 & 135 & $150.3 \pm 1.6$ \\
\hline
\end{tabular}

Table 6: $\rho$ decay widths.

We also note the following relation between couplings of the axial resonances defined in the lagrangian (C.1) of Appendix O,

$$
\alpha_{A_{n}}=-\frac{1}{2 \sqrt{2}} f_{A_{n}} .
$$

Relation (5.4) implies the vanishing of the coupling of any axial resonance $A^{(n)}$ to one pion and a vector source/photon [52]. The decay $a_{1} \rightarrow \pi \gamma$ does not occur at this order in the

\footnotetext{
${ }^{7}$ The constants $f_{V_{n}}$, defined via $\left\langle 0\left|J_{V}^{\mu}(0)\right| V^{(n)}(\varepsilon, p)\right\rangle=\varepsilon^{\mu} f_{V_{n}} M_{V_{n}}^{2}$ describe the decay of the mesons into leptons when the electroweak sector is coupled to the QCD currents. $g_{V_{n}}$ describes the coupling of vector mesons to two pions.
} 
minimal model. (The same is true for all axial resonances $a_{1}^{\prime}, a_{1}^{\prime \prime}, \ldots$.) Higher-derivative terms would modify this result, but should only be considered at higher orders. In Section 7.4, we will introduce modifications of the metric that are felt differently by the axial and vector combinations of gauge fields (in order to account for local order-parameters of chiral symmetry). We plan to investigate how this modifies $\Gamma\left(a_{1} \rightarrow \pi \gamma\right)$ in a forthcoming paper.

To illustrate how the currents (quark bilinears in QCD) are expressed in terms of mesons, we use the expressions provided in the Appendix $\mathrm{Q}$. One can then derive a fieldcurrent identity [53]

$$
J_{V \mu}^{a}(x)=\sum_{n=1}^{\infty} f_{V n} M_{V n}^{2} V_{\mu}^{a(n)}(x)+\text { non-linear terms }
$$

with the expressions for the decay constants given by ${ }^{8}$

$$
\eta^{\mu \nu} f_{V_{n}} M_{V_{n}}^{2}=-\left.\frac{\sqrt{2}}{g_{5}^{2}} \sqrt{g} g^{\mu \nu} g^{55} \partial_{5} \varphi_{n}^{V}\right|_{l_{0}}
$$

Similarly, we get the corresponding formula for the axial current

$$
J_{A \mu}^{a}(x)=f \partial_{\mu} \pi^{a}+\sum_{n=1}^{\infty} f_{A n} M_{A_{n}}^{2} A_{\mu}^{a(n)}(x)+\text { non-linear terms }
$$

\section{High energies: sums of resonance contributions}

In this Section, we examine the following amplitudes: $\left\langle\pi\left|J_{V}^{\mu}\right| \pi\right\rangle,\left\langle\gamma\left|J_{A}^{\mu}\right| \pi\right\rangle$. The Lorentzinvariant functions involved are called vector form factor and axial form factor. We also consider forward elastic scattering of GBs $\langle\pi \pi \mid \pi \pi\rangle$. We discuss how many subtractions are needed for the corresponding dispersion relations to converge.

Our interest in these questions is motivated by the precedent of $4 \mathrm{D}$ models with a finite number of resonance multiplets. In this case, one imposed that vector form factor, axial form factor and GB forward-scattering amplitudes satisfy dispersion relations with an appropriate number of subtraction (respectively 0,0 and 1). This soft high-energy behavior is the one expected from QCD [11]. This also brought the different formalisms for massive spin-1 fields into agreement.

\subsection{Sums over resonance couplings}

Before proceeding, we make the following remark, of use for the present Section. In our

\footnotetext{
${ }^{8} \mathrm{Such}$ a result can also be understood from a more holographic point of view, by plugging in the KK decomposition in equation (6) of 33].
} 
$5 \mathrm{D}$ model, we automatically obtain the following sum rules

$$
\begin{aligned}
\sum_{n=1}^{\infty} f_{V_{n}} g_{V_{n}} & =2 L_{9}, \\
\sum_{n=1}^{\infty} f_{V_{n}} g_{V_{n}} M_{V_{n}}^{2} & =f^{2}, \\
\sum_{n=1}^{\infty} g_{V_{n}}^{2} & =8 L_{1}, \\
\sum_{n=1}^{\infty} g_{V_{n}}^{2} M_{V_{n}}^{2} & =\frac{f^{2}}{3} .
\end{aligned}
$$

They are derived using the completeness relation for the KK wave-functions. One starts from the explicit expression for the resonance couplings of Appendix $\mathbb{\mathrm { G }}$, and uses as well the EOMs and BCs for the various wave-functions. The sum rules are thus a consequence of the gauge symmetry and the 5D locality. The expressions (4.6, 4.9, 4.10) for the low-energy constants $f, L_{1}, L_{9}$ appearing in the right-hand side of (6.1-6.4) are finite for the metrics of interest. Convergence can be checked explicitly for flat space and RS1, using the behavior with $n$ of couplings and masses, Tables 3 and 5 .

The relations (6.1-6.3) will look familiar from 4D models of resonances: in that case, one had finite sums rather than infinite ones. One required relations such as $(6.1-6.3)$ in order to guarantee the QCD behavior at high energies [11. Certain 4D models provide some of these relations automatically. In this Section, we explore the consequences of 6.1-6.3) on the high-energy behavior of some amplitudes.

\subsection{Vector form factor}

The vector form factor $F\left(q^{2}\right)$ is defined as $\left(q=p-p^{\prime}\right)$

$$
\left\langle\pi^{a}\left(p^{\prime}\right)\left|J_{V}^{b \mu}(0)\right| \pi^{c}(p)\right\rangle=\mathrm{i} \varepsilon^{a b c}\left(p+p^{\prime}\right)^{\mu} F\left(q^{2}\right) .
$$

\subsubsection{D point of view}

In this Section, we describe the vector form factor in terms of exchange of (an infinite number) of resonances. From a partonic analysis [54, 55], it is expected that the vector form factor satisfies an unsubtracted dispersion relation. We show that such a soft behavior is satisfied in our model.

The vector form factor gets contributions from the $\mathcal{O}\left(p^{2}\right)$ term in the chiral lagrangian (4.5), the $L_{9}$ term in the $\mathcal{O}\left(p^{4}\right)$ Lagrangian, and the exchange of resonances

$$
F\left(q^{2}\right)=1+2 L_{9} \frac{q^{2}}{f^{2}}+\frac{q^{4}}{f^{2}} \sum_{n=1}^{\infty} \frac{f_{V_{n}} g_{V_{n}}}{M_{V_{n}}^{2}-q^{2}} .
$$

This expression is convenient for low energies. The high-energy behavior of the infinite sum (6.6) however, is not completely transparent. This is because it takes the form of a 
twice-subtracted dispersion relation for $F\left(q^{2}\right)$. Indeed, reading off the imaginary part of $F\left(q^{2}\right)$ from $(6.6)$

$$
\frac{1}{\pi} \operatorname{Im} F(s)=\frac{1}{f^{2}} \sum_{n=1}^{\infty} f_{V_{n}} g_{V_{n}} M_{V_{n}}^{4} \delta\left(s-M_{V_{n}}^{2}\right)
$$

(6.6) is equivalent to $F\left(q^{2}\right)=F(0)+F^{\prime}(0) q^{2}+\frac{q^{4}}{\pi} \int_{0}^{\infty} \frac{\mathrm{d} s}{s^{2}} \frac{\operatorname{Im} F(s)}{s-q^{2}}$. As is usual in large- $N_{c}$ approximations, the dispersive integral has converted to a discrete sum in (6.6), which converges as long as $\sum_{n} f_{V_{n}} g_{V_{n}} / M_{V_{n}}^{2}$ converges. However, we can do better than that: since the sum $\sum_{n} f_{V_{n}} g_{V_{n}} M_{V_{n}}^{2}$ converges (Table 5), one finds that the two subtractions are unnecessary. Using the values for the sums $\sum_{n} f_{V_{n}} g_{V_{n}}=2 L_{9}$ (6.1) and $\sum_{n} f_{V_{n}} g_{V_{n}} M_{V_{n}}^{2}=f^{2}$ (6.2), one obtains

$$
F\left(q^{2}\right)=\frac{1}{f^{2}} \sum_{n} f_{V_{n}} g_{V_{n}} \frac{M_{V_{n}}^{4}}{M_{V_{n}}^{2}-q^{2}},
$$

which is an unsubtracted dispersion relation (c.f. the imaginary part (6.7)).

We emphasize that the result obtained above, i.e. that the vector form factor satisfies an unsubtracted dispersion relation, does not depend on the metric. Similar relations are not automatic in 4D models for resonances: even in hidden local symmetry models, it needs to be imposed by fixing one free parameter. There is therefore a non-trivial step in the high-energy behavior in going from hidden local symmetry to a 5D model. The possibility of rewriting the vector form factor as a sum of poles without contact terms is indeed specific of a continuous extra-dimension, as the case of a discrete extra-dimension [19] demonstrated. The unsubtracted dispersion relation then follows.

\subsubsection{D point of view}

Using the 5D language, one obtains from the lagrangian

$$
f^{2} F\left(q^{2}\right)=f^{2}+\frac{q^{2}}{2 g_{5}^{2}} \int_{l_{0}}^{l_{1}} \mathrm{~d} z w(z) \Gamma(z)+\frac{q^{4}}{2 g_{5}^{4}} \int_{l_{0}}^{l_{1}} \mathrm{~d} z w(z) \Gamma(z) \int_{l_{0}}^{l_{1}} \mathrm{~d} z^{\prime} w\left(z^{\prime}\right) G_{q}\left(z, z^{\prime}\lfloor 6.9)\right.
$$

with $\Gamma(z) \equiv 1-\alpha(z)^{2}$. This expression is the $5 \mathrm{D}$ version of (6.6). The $5 \mathrm{D}$ propagator occurring above is the mixed position-momentum propagator $G_{q}\left(z, z^{\prime}\right)$ defined as

$$
G\left(x, z ; x^{\prime}, z^{\prime}\right)=\int \frac{\mathrm{d}^{4} p}{(2 \pi)^{4}} \mathrm{e}^{\mathrm{i} q \cdot\left(x-x^{\prime}\right)} G_{q}\left(z, z^{\prime}\right) .
$$

It satisfies

$$
\left\{q^{2}+\frac{1}{w(z)} \partial_{z}\left(w(z) \partial_{z}\right)\right\} G_{q}\left(z, z^{\prime}\right)=-\frac{g_{5}^{2}}{w(z)} \delta\left(z-z^{\prime}\right)
$$

The relation with expression (6.6) is provided by the KK decomposition of the 5D propagator

$$
G_{q}\left(z, z^{\prime}\right)=\sum_{n=1}^{\infty} \frac{\varphi_{n}^{V}(z) \varphi_{n}^{V}\left(z^{\prime}\right)}{M_{V_{n}}^{2}-q^{2}}
$$


and the expressions for the resonance couplings $f_{V_{n}}, g_{V_{n}}$ of Appendix Q. In 5D language, one can obtain a more compact expression for the vector form factor by using the EOM for the $5 \mathrm{D}$ propagator (6.11). We get

$$
f^{2} F\left(q^{2}\right)=f^{2}+\left.\frac{q^{2}}{2 g_{5}^{4}} \int_{l_{0}}^{l_{1}} \mathrm{~d} z w(z) \Gamma(z) \partial_{z^{\prime}} G_{q}\left(z, z^{\prime}\right)\right|_{z^{\prime}=l_{0}},
$$

where the pure $L_{9}$ term has disappeared. We have used the normalization $w\left(z^{\prime}=l_{0}\right)=1$. Finally, one can use again the EOM to absorb the contact term and obtain a compact form

$$
f^{2} F\left(q^{2}\right)=\left.\frac{1}{2 g_{5}^{4}} \int_{l_{0}}^{l_{1}} \mathrm{~d} z w(z)\left\{\partial_{z} \Gamma(z)\right\} \partial_{z} \partial_{z^{\prime}} G_{q}\left(z, z^{\prime}\right)\right|_{z^{\prime}=l_{0}} .
$$

This expression coincides with the 4D one (6.8).

During this section and the next one, we will use schematic drawings to represent this kind of relationships, replacing for instance $\int \mathrm{d} z^{\prime} w\left(z^{\prime}\right)$ by $\int^{\prime}$, and indicating the 5D propagator by a double line, and sources by crosses. The three contributions of equation (6.9) are shown schematically in the first line of (6.15), and the compact result (6.14) is depicted in the last line of (6.15).

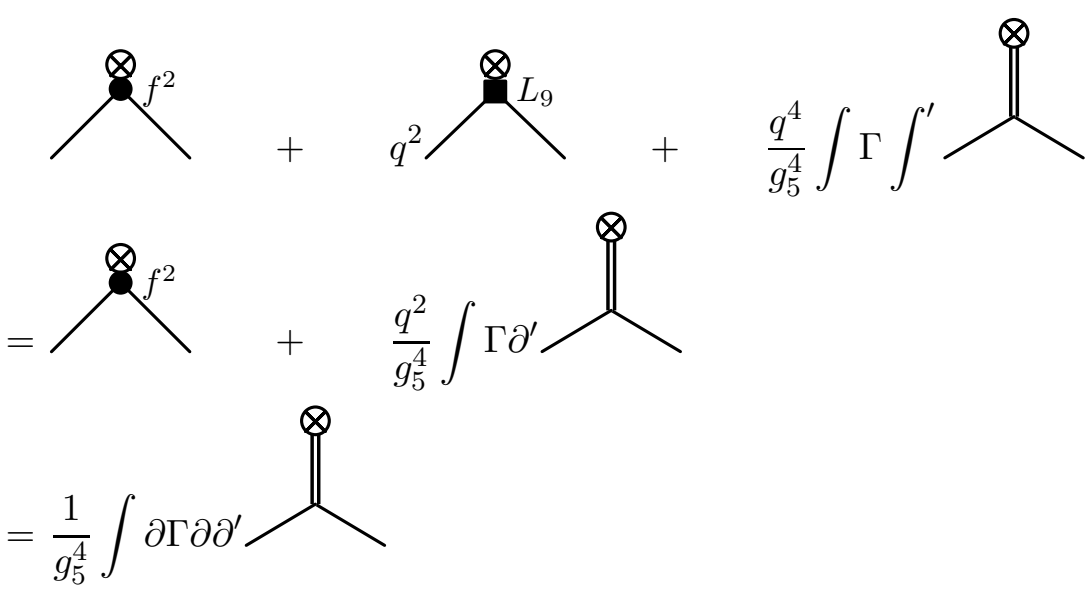

\subsection{Froissart bound}

Having discussed in detail the unsubtracted dispersion relation for the vector form factor, we briefly give the result for GB scattering. The reasoning parallels that of [11], since we have the relations $L_{2}=-2 L_{1}(4.12)$ and $L_{3}=-6 L_{1}(4.13){ }^{9}$. We also have the relation $\sum_{n} g_{V_{n}}^{2}=8 L_{1}$ (6.3), which generalizes the usual one $g_{\rho}^{2}=8 L_{1}$ to an infinite sum.

One considers forward $(t=0)$ elastic GB scattering, which receives contributions from the $\chi \mathrm{PT}$ lagrangian, through the constants $L_{1}, L_{2}, L_{3}$, and from resonance exchange. Combining left- and right-handed cuts using the variable $\nu \equiv(s-u) / 2$, and using the relations just mentioned, one shows that the scattering amplitude satisfies a once-subtracted dispersion relation, as expected from the Froissart bound [56]. Indeed, the elastic forward scattering amplitude can be rewritten as

$$
T_{i j}(\nu)=T_{i j}(0)+\kappa_{i j} \frac{\nu^{2}}{f^{4}} \sum_{n=1}^{\infty} g_{V_{n}}^{2} \frac{M_{V_{n}}^{4}}{M_{V_{n}}^{4}-\nu^{2}},
$$

\footnotetext{
${ }^{9}$ These relations were derived assuming $N_{f}=3$ : we stick to this case here.
} 
where $i, j$ are $\mathrm{SU}\left(N_{f}=3\right)$ quantum numbers and $\kappa_{i j}$ is a constant depending on the process. The subtraction constant $T_{i j}(0)$ vanishes for GBs, from Adler's theorem.

\subsection{Axial form factor}

The definition of the axial form factor $G_{A}\left(q^{2}\right)$ is $\left(q=p-p^{\prime}\right)$

$$
\left\langle\gamma\left(\epsilon, p^{\prime}\right)\left|J_{A}^{a \mu}(0)\right| \pi^{b}(p)\right\rangle=\sqrt{2} \frac{e}{f}\left(p^{\prime \mu} p \cdot \epsilon-\epsilon^{\mu} p \cdot p^{\prime}\right) G_{A}\left(q^{2}\right)+\text { pion pole term. }(6.17)
$$

Our model yields the following generalization of the result obtained in [52]

$$
G_{A}\left(q^{2}\right)=4\left(L_{9}+L_{10}\right)+q^{2} \sum_{n=1}^{\infty} \frac{f_{A_{n}}\left(f_{A_{n}}+2 \sqrt{2} \alpha_{A_{n}}\right)}{M_{A_{n}}^{2}-q^{2}} .
$$

Plugging in the relations that hold in our model (independently of the metric), i.e. $L_{9}+$ $L_{10}=0$ (4.14) and $f_{A_{n}}=-2 \sqrt{2} \alpha_{A_{n}}$ (5.4), we find that the axial form factor vanishes identically

$$
G_{A}\left(q^{2}\right)=0
$$

Including higher-derivative terms in the action would produce a non-vanishing resonanceexchange contribution. This is however of higher-order in the presumed derivative expansion. In our 5D model, the contact term $4\left(L_{9}+L_{10}\right)$ in (6.18) vanishes, which is the only way that $G_{A}\left(q^{2}\right)$ can satisfy an unsubtracted dispersion relation, given the vanishing of resonance-exchange terms. Therefore, the axial form factor in our $5 \mathrm{D}$ model satisfies an unsubtracted dispersion relation, albeit in a trivial way. Modifications of the metric in the IR, if they are felt differently by vector and axial combinations as introduced later in Section 7.4, will change this. We plan to investigate this in future work.

\subsection{Lightest vector-meson dominance}

For completeness, we discuss vector-meson dominance and KSFR I \& II relations. The fact that the KSFR relations are not obeyed in the model leaves room for better experimental agreement, see Table 6 .

The sum rules (6.1-6.3) constitute a generalization of the relations considered in 11] in the sense that, on the resonance (i.e. left-hand) side of equations, the sum involves an infinite number of terms. In the 5D model, the sum rules hold for any metric, but the relative contribution of each resonance $\rho, \rho^{\prime}, \rho^{\prime \prime}, \cdots$ depends on the metric. We ask to what extent the sums are satisfactorily reproduced by taking into account only the $\rho$, i.e. to which extent the lightest meson dominates the sum, or the statement of lightest vector-meson dominance (LMD), see Table 7. We see that the sums indeed receive a large contribution from the $\rho$, for both metrics ${ }^{10}$.

Related to the last line in Table 7 is the question of the KSFR II relation. Within our notations, the KSFR II relation would be the statement that the definition

$$
\frac{1}{a} \equiv \frac{g_{V_{1}}^{2} M_{V_{1}}^{2}}{f^{2}}
$$

\footnotetext{
${ }^{10}$ This is true for sums involving vector resonances only. On the other hand, saturation by $\rho$ and $a_{1}$ does not hold for sums such as $\sum_{n}\left(f_{V_{n}}^{2} M_{V_{n}}^{2 k}-f_{A_{n}}^{2} M_{A_{n}}^{2 k}\right)$, i.e. moments of the left-right correlator such as $L_{10}$ and the Weinberg sum rules, see Appendix $D$.
} 
would yield $a=2$. In the $5 \mathrm{D}$ model, one sees from the sum rule $\sum_{n} g_{V_{n}}^{2} M_{V_{n}}^{2}=f^{2} / 3$ (6.4) that the natural value for the KSFR II ratio is 3 rather than 2. Indeed, the sum (6.4) is dominated by the contribution from the $\rho$ (Table 7), and the KSFR II ratio takes a value close to 3 , as already noted in [34]. Regarding the KSFR I relation $f_{\rho}=2 g_{\rho}$, the only related sum rule we find in our model is $\sum_{n=1}^{\infty}\left(2 f_{V_{n}} g_{V_{n}}-f_{V_{n}}^{2}+f_{A_{n}}^{2}\right)=4\left(L_{9}+L_{10}\right)=0$ : the axial resonance contribution cannot be separated. There is therefore no favored value for the KSFR I ratio: it will depend on the metric.

\section{High energies: two-point functions and OPE}

In this section we compute the two-point functions $\Pi_{V, A}$ in the RS1 case. The RS1 metric indeed has the right behavior near the UV brane to yield the partonic logarithm in the OPE of $\Pi_{V, A}(2.9)$. This is discussed below.

We will first derive compact expressions for $\Pi_{V, A}$ following the rules of $5 \mathrm{D}$. We recall that the $5 \mathrm{D}$ treatment preserves the full symmetry

\begin{tabular}{|c|c|c|}
\hline & $w(z)=1$ & $w(z)=\frac{l_{0}}{z}$ \\
\hline$\frac{f_{V_{1}} g_{V_{1}}}{\sum_{n} f_{V_{n}} g_{V_{n}}}$ & 0.99 & 1.02 \\
\hline$\frac{f_{V_{1}} g_{V_{1}} M_{V_{1}}^{2}}{\sum_{n} f_{V_{n}} g_{V_{n}} M_{V_{n}}^{2}}$ & 0.81 & 1.11 \\
\hline$\frac{g_{V_{1}}^{2}}{\sum_{n} g_{V_{n}}^{2}}$ & 0.999 & 0.999 \\
\hline$\frac{g_{V_{1}}^{2} M_{V_{1}}^{2}}{\sum_{n} g_{V_{n}}^{2} M_{V_{n}}^{2}}$ & 0.99 & 0.99 \\
\hline
\end{tabular}

Table 7: Relative contributions of the $\rho$ resonance as compared to the total sums of Section 6.1. structure of the model and is the suitable one for high energies. In Appendix $\mathrm{D}$, we discuss sum rules related to $\Pi_{L R}$ from the resonance/4D point of view: the infinite sums over resonances need to be regularized. The two pictures are related by writing the $5 \mathrm{D}$ propagator $G_{q}\left(z, z^{\prime}\right)$ as a sum of resonance poles (6.12).

\subsection{The vector and axial two-point functions}

The vector two-point function is obtained by taking two derivatives with respect to sources in the generating functional. This leads to contact contributions coming from the $\mathcal{O}\left(p^{4}\right)$ lagrangian and resonance exchange terms, which can be expressed directly in terms of the $5 \mathrm{D}$ propagator

$$
\Pi_{V}\left(q^{2}\right)=-4 L_{10}-8 H_{1}+2 \frac{q^{2}}{g_{5}^{4}} \int_{l_{0}}^{l_{1}} \mathrm{~d} z w(z) \int_{l_{0}}^{l_{1}} \mathrm{~d} z^{\prime} w\left(z^{\prime}\right) G_{q}\left(z, z^{\prime}\right) .
$$

We can use the equation of motion of the $5 \mathrm{D}$ propagator (6.11), and the expressions of $L_{10}$ (4.10) and $H_{1}$ (4.11) to rewrite the two-point function as

$$
\Pi_{V}\left(q^{2}\right)=\left.\frac{2}{q^{2} g_{5}^{4}} \partial_{z} \partial_{z^{\prime}} G_{q}\left(z, z^{\prime}\right)\right|_{z=z^{\prime}=l_{0}} .
$$

In the above, we have assumed the normalization $w\left(z=l_{0}\right)=1$ for the warp factor at the UV brane. Going from equation (7.1) to (7.2) is schematized as

$$
\begin{aligned}
\Pi_{V} & =\stackrel{H_{1}, L_{10}}{\otimes} \otimes \frac{q^{2}}{g_{5}^{4}} \iint^{\prime} \otimes \stackrel{V}{=} \otimes \\
& =\left.\frac{1}{q^{2} g_{5}^{4}} \partial \partial^{\prime} \otimes \stackrel{V}{=} \otimes\right|_{l_{0}, l_{0}} .
\end{aligned}
$$


In the first expression, the contact terms appear explicitly outside the integral involving the $5 \mathrm{D}$ resonance field propagator. In the final expression, only the second derivative of the $5 \mathrm{D}$ propagator evaluated at the boundary appears. This embodies the idea of holography: one could have used a completely equivalent way of obtaining the two-point function: by using directly the field-current identity (5.5) in the expression for the two-point function (2.8). This directly yields the "holographic-looking" result (7.2).

The same remarks hold for the axial case, in which case there are contributions from the $\mathcal{O}\left(p^{2}\right)$ lagrangian (4.5) via a pion pole and a contact term, yielding a transverse structure. We write, with the pion propagator represented by a simple line

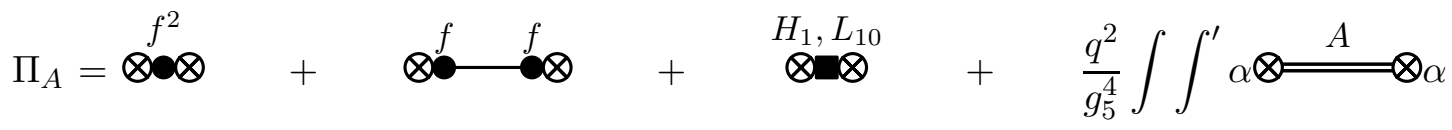

$$
\begin{aligned}
& =\left.\frac{1}{q^{2} g_{5}^{4}} \partial \partial^{\prime} \otimes \stackrel{A}{=} \otimes\right|_{l_{0}, l_{0}} .
\end{aligned}
$$

As depicted above, the compact expression obtained after using twice the EOM for the 5D propagator (6.11) includes the pion pole term. This is due to the BCs for $\alpha$ and for $A_{\mu}$.

\subsubsection{Explicit expression for RS1}

In this Section, we specialize to the case of RS1. The propagator in RS1 satisfies the EOM (6.11) with $w(z)=l_{0} / z$, i.e.

$$
\left\{q^{2}+z \partial_{z}\left(\frac{1}{z} \partial_{z}\right)\right\} G_{q}\left(z, z^{\prime}\right)=-g_{4}^{2} z \delta\left(z-z^{\prime}\right) .
$$

This leads to the explicit form involving Bessel functions

$$
G_{q}\left(z, z^{\prime}\right)=-\frac{g_{4}^{2} z z^{\prime}}{\alpha-\beta}\left\{I_{1}\left(Q z_{>}\right)-\alpha K_{1}\left(Q z_{>}\right)\right\}\left\{I_{1}\left(Q z_{<}\right)-\beta K_{1}\left(Q z_{<}\right)\right\}
$$

where $z_{<,>}=\min (\max )\left(z, z^{\prime}\right)$. Depending on the boundary conditions $(+$ or -$)$, the values of $\alpha$ and $\beta$ are given by

$$
\begin{aligned}
& \mathrm{BC}+/- \text { at } l_{1}: \alpha_{ \pm}=\frac{-I_{0}\left(Q l_{1}\right)}{K_{0}\left(Q l_{1}\right)}, \frac{I_{1}\left(Q l_{1}\right)}{K_{1}\left(Q l_{1}\right)}, \\
& \mathrm{BC}+/- \text { at } l_{0}: \beta_{ \pm}=\frac{-I_{0}\left(Q l_{0}\right)}{K_{0}\left(Q l_{0}\right)}, \frac{I_{1}\left(Q l_{0}\right)}{K_{1}\left(Q l_{0}\right)} .
\end{aligned}
$$

After simplifications, the two-point functions $\Pi_{V, A}$ exhibit quite compact expressions

$$
\Pi_{V, A}\left(-Q^{2}\right)=\frac{2}{g_{4}^{2}} \frac{\left\{I_{0}\left(Q l_{0}\right)+\alpha_{ \pm} K_{0}\left(Q l_{0}\right)\right\}\left\{I_{0}\left(Q l_{0}\right)+\beta_{-} K_{0}\left(Q l_{0}\right)\right\}}{\alpha_{ \pm}-\beta_{-}} .
$$

We now assume that the momentum involved remains below $1 / l_{0}$, and perform an expansion in terms of $Q l_{0}$,

$$
\Pi_{V, A}\left(-Q^{2}\right)=\frac{-1}{g_{4}^{2}}\left(\log \frac{Q^{2}}{\mu^{2}}+\lambda_{\mathrm{UV}}(\mu)\right)+\frac{2}{g_{4}^{2} \alpha_{ \pm}\left(Q l_{1}\right)}+\mathcal{O}\left(Q^{2} l_{0}^{2}\right),
$$


where $\lambda_{\mathrm{UV}}(\mu)=\log \left(\mu^{2} l_{0}^{2} / 4\right)+2 \gamma_{E}$. We have introduced a renormalization scale $\mu$, independent of the regulator $1 / l_{0}$. The expression (7.10) is valid for all energies below the regulator $Q \ll 1 / l_{0}$, whether $Q$ is below $M_{\rho} \sim 2.4 / l_{1}$ or above it.

The $\Pi_{V, A}$ describe Green's functions of operators which, in QCD, are composite (quark bilinears). Nonetheless, one can send the regulator $l_{0} \longrightarrow 0$ and still get finite results for $\Pi_{V, A}$, provided a counter-term $\delta\left(z-l_{0}\right) H_{1}^{\text {brane }}\left\langle R_{\mu \nu} R_{\mu \nu}+L_{\mu \nu} L_{\mu \nu}\right\rangle$ is added to the 5D YM lagrangian (2.3). Such a term is allowed by the symmetries of the model and yields, after the field redefinitions of Section 3 , an additional contribution to the source contact term $H_{1}$ (4.11). If the coefficient $H_{1}^{\text {brane }}$ depends on $l_{0}$ so as to compensate the divergence of (7.10) when $l_{0} \longrightarrow 0$, this allows us to remove the regulator $l_{0} \longrightarrow 0$ in $(7.10)$, and consider arbitrarily large momenta. In the following we always assume that momenta are smaller than the regulator $1 / l_{0}$, or that the regulator is sent to infinity.

Comparing with the QCD OPE expansion (2.9), we identify the combination of parameters of the 5D RS1 model that correspond to $N_{c}$ [19]

$$
N_{c}=\frac{12 \pi^{2}}{g_{4}^{2}}
$$

We can now go back to the expressions of Section 4 and 5 and check the large- $N_{c}$ scaling of quantities like the pion decay constant, the $L_{i}$ constants, as well as the resonance masses and couplings. We find as expected from [1, 2, 5]

$$
\begin{array}{r}
f^{2}, L_{1}, L_{2}, L_{3}, L_{9}, L_{10}, f_{V_{n}}, g_{V_{n}}, f_{A_{n}} \\
M_{V_{n}}, M_{A_{n}}
\end{array}
$$

Using as inputs $f_{\pi}=87 \mathrm{MeV}$ and $M_{\rho}=776 \mathrm{MeV}$, we find $N_{c} \simeq 4.3$ instead of 3 . This is the reason why we have used $M_{\rho}$ rather than $N_{c}$ as an input to give numerical values for low-energy data. The order of magnitude for $N_{c}$ is right, but the situation may still be improved by taking into account deformations of the metric, as we suggest in Section 7.4. This would take into account condensates, not limited to $\langle\bar{q} q\rangle$ and without introducing the additional degrees of freedom of [33, 34].

The second piece in the correlator $(7.10)$ is the spontaneous symmetry breaking produced by different BCs in the IR

$$
\text { for } Q \gg 1 / l_{1}, \quad \frac{1}{\alpha_{ \pm}} \simeq \mp \pi e^{-2 Q l_{1}} \text {. }
$$

From the 5D point of view the interpretation of this term is clear: it is caused by the breaking of conformal invariance due to the finite size of a compact extra-dimension. This breaking will be manifest only at energies below the compactification scale, the scale of the first resonance [57]. The fact that vector and axial fields feel different BCs in the IR will produce a nonzero value of the left-right correlator at low energies but exponentially going to zero at high ernergies. Interestingly, (7.14 adopts the form of a violation of the quark-hadron duality by a finite size instanton [13]. 
To see how the two-point function $\Pi_{V}$ contains already an infinite number of resonances, let us write it for time-like momenta $q^{2}>0$

$$
g_{4}^{2} \Pi_{V}\left(q^{2}\right)=-\lambda_{\mathrm{UV}}(\mu)-\log \left(\frac{q^{2}}{\mu^{2}}\right)+\pi \frac{Y_{0}\left(q l_{1}\right)}{J_{0}\left(q l_{1}\right)} .
$$

Taking the high-energy limit $q l_{1} \gg 1$, one sees explicitly the two contributions: the logarithm and the sum over an infinite number of resonances

$$
\text { for } q \gg 1 / l_{1}, \quad g_{4}^{2} \Pi_{V}\left(q^{2}\right) \simeq-\lambda_{\mathrm{UV}}(\mu)-\log \left(\frac{q^{2}}{\mu^{2}}\right)+\tan \left(q l_{1}-\frac{\pi}{4}\right) .
$$

At low energies $q l_{1} \ll 1$, the logarithmic contribution is exactly canceled by part of the second term in $(7.15)$ to end up with

$$
\text { for } q \ll 1 / l_{1}, \quad g_{4}^{2} \Pi_{V}\left(q^{2}\right) \simeq 2 \log \left(\frac{l_{1}}{l_{0}}\right)+\frac{1}{2}\left(q l_{1}\right)^{2}+\frac{5}{64}\left(q l_{1}\right)^{4}+\mathcal{O}\left(q^{6} l_{1}^{6}\right) .
$$

Note that the constant term is the expected one since, in RS1, we have $2 \log \left(l_{1} / l_{0}\right)=$ $-4 L_{10}-8 H_{1}$. Again, the limit $l_{0} \longrightarrow 0$ can be taken if the additional contribution $H_{1}^{\text {brane }}\left(l_{0}\right)$ is considered.

\subsection{The left-right correlator}

From the result for $\Pi_{V, A}(7.10)$, we see that the left-right correlator in this model is described in terms of Bessel functions, that are valid for any energy below the UV regulator $1 / l_{0}$

$$
\Pi_{L R}\left(-Q^{2}\right) \equiv \frac{1}{2}\left(\Pi_{V}\left(-Q^{2}\right)-\Pi_{A}\left(-Q^{2}\right)\right)=\frac{-1}{g_{4}^{2} Q l_{1}} \frac{1}{I_{0}\left(Q l_{1}\right) I_{1}\left(Q l_{1}\right)}+\mathcal{O}\left(Q l_{0}\right)
$$

We can explore two regimes, low and high energies, depending on how the momentum is compared with the resonance mass scale, $1 / l_{1}$. For large euclidean momentum

$$
\text { for } Q \gg 1 / l_{1}, \quad \Pi_{L R}\left(Q^{2}\right) \simeq \frac{-2}{g_{4}^{2}} \pi \mathrm{e}^{-2 Q l_{1}} .
$$

For large $Q$, the left-right correlation function vanishes faster than any power of $Q^{2}$

$$
\lim _{Q^{2} \rightarrow \infty} Q^{2 n} \Pi_{L R}\left(Q^{2}\right)=0, \quad \text { for all } n .
$$

This means that no local order parameter appears in its OPE expansion. In particular, the dimension three condensate $\langle\bar{q} q\rangle$ is absent [29]. The exponential fall-off at high energies [28] is readily understood in the model, since the symmetry is broken on the IR brane, at a finite distance from us in the fifth dimension, and therefore generates only non-local order parameters. We will see how this translates in terms of resonance parameters in Appendix D, yielding Weinberg sum rules.

For very low momentum, $Q \ll 1 / l_{1}$,

$$
\text { for } Q \ll 1 / l_{1}, \quad \Pi_{L R}\left(Q^{2}\right) \simeq \frac{1}{g_{4}^{2}}\left(-\frac{2}{\left(Q l_{1}\right)^{2}}+\frac{3}{4}-\frac{17}{96}\left(Q l_{1}\right)^{2}\right)+\mathcal{O}\left(Q^{4} l_{1}^{4}\right) .
$$

This expression takes the standard form expected from $\chi \mathrm{PT}$ with large- $N_{c}: \Pi_{L R}\left(Q^{2}\right)=$ $-f_{\pi}^{2} / Q^{2}-4 L_{10}+\mathcal{O}\left(Q^{2}\right)$ : we recover the values for $f$ and $L_{10}$ obtained in (4.7) and Table 1. 


\section{$7.34 \mathrm{D}$ point of view}

The above results for the two-point correlators were derived directly using the 5D propagator. It is a general fact in our model that such compact expressions can be derived in the 5D picture. On the other hand, the imaginary part of the Green's function involves sums over KK modes, hiding the 5D origin of the model: in any model with narrow resonances, the imaginary part of $\Pi_{V}(s)$ is given by

$$
\frac{1}{\pi} \operatorname{Im} \Pi_{V}(s)=2 \sum_{n=1}^{\infty} f_{V_{n}}^{2} M_{V_{n}}^{2} \delta\left(s-M_{V_{n}}^{2}\right) .
$$

Indeed, the $4 \mathrm{D}$ version of equation (7.1) is

$$
\Pi_{V}\left(q^{2}\right)=-4 L_{10}-8 H_{1}-q^{2} \sum_{n=1}^{\infty} \frac{f_{V_{n}}^{2}}{M_{V_{n}}^{2}-q^{2}} .
$$

The equivalence between the two writings (7.23) and (7.1) is provided by the relation between $5 \mathrm{D}$ and $4 \mathrm{D}$ propagators, equation (6.12). Notice that $(7.23)$ can be seen as a once-subtracted dispersion relation obtained from (7.22).

Surely, an expression such as (7.22) cannot reproduce the partonic result that $\operatorname{Im} \Pi_{V}$ tends to a constant for large time-like momenta $\frac{1}{\pi} \operatorname{Im} \Pi_{V}^{\mathrm{QCD}}(s) \underset{s \rightarrow \infty}{\longrightarrow} \frac{N_{c}}{12 \pi^{2}}$. However, a sum over an infinite number of terms can reproduce the right logarithmic behavior for large euclidean momenta $Q^{2} \longrightarrow+\infty$, provided the decay constants for the heavy resonances

follow the power-law $f_{V_{n}}^{2} \underset{n \rightarrow \infty}{\propto} \frac{1}{n}$ [58, 16]. A look at Table 5 shows that the RS1 metric yields the right behavior in the limit $l_{0} \longrightarrow 0$. Note that one can derive the sum rule

$$
\sum_{n=1}^{\infty} f_{V_{n}}^{2}=\frac{1}{g_{5}^{2}} \int_{l_{0}}^{l_{1}} \mathrm{~d} z w(z)
$$

using the completeness relation as before. For RS1, we have $\frac{w(z)}{g_{5}^{2}}=\frac{1}{g_{4}^{2} z}$ : the divergence at the UV boundary of the integral in (7.24) when $l_{0} \longrightarrow 0$ is related to the $1 / n$ behavior for the $f_{V_{n}}^{2}$. For $l_{0}>0$, the integral (7.24) does not diverge. In this case, we have seen in (7.10) that the logarithmic behavior was reproduced for $Q \ll 1 / l_{0}$, with corrections analytic in $Q l_{0}$.

We can now turn back to our once-subtracted dispersion relation for $\Pi_{V}$ (7.23). We understand that the subtraction is necessary in the limit $l_{0} \longrightarrow 0$ for RS1. However, we have seen that the 5D expression corresponding to this once-subtracted dispersion relation (7.1) could be recast in a more compact form (7.2). This is so because the $5 \mathrm{D}$ description is not encumbered by the infinite sums which may diverge. In 5D, one gets analytic expressions: discussing the number of subtractions is then replaced by considering the behavior at large momenta, which can be done directly as in (7.10) for euclidean momenta, or as (7.15) for $q^{2}>0$. This is to be contrasted with the $4 \mathrm{D}$ infinite sums, for which the limit $q^{2} \longrightarrow+\infty$ is hampered by the presence of an infinite number of resonances with masses $M^{2}>q^{2}$ for any given $q^{2}$. From the 5D expressions, the limit $\left|q^{2}\right| \longrightarrow \infty$ is easily obtained: provided one stays below $1 / l_{0}$ or sends it to infinity, the only scale to compare the momentum to is $1 / l_{1}$ which fixes all resonance masses at once. 


\subsection{Condensates}

Given the breaking by BCs and the exact conformality of the metric, broken only by the IR brane, the model we have presented here has no local order parameters. However, any metric that is asymptotically AdS near the UV brane

$$
w(z) \underset{z \rightarrow l_{0}}{\sim} \frac{l_{0}}{z}
$$

will produce the dimension zero term (2.9) in the OPE of $\Pi_{V, A}$. More generally, one can introduce deformations of conformality in the bulk, which will reproduce the condensates. In [33, 34], deformations were introduced by a 5D scalar field obtaining a vacuum expectation value. Here, we wish to keep the field content minimal: we introduce deformations of the metric: as was the case for the BCs, the deformations should be felt differently by the axial and vector combinations of fields

$$
w_{V, A}(z)^{2}=\left(\frac{l_{0}}{z}\right)^{2}\left(1+\sum_{d=2} c_{d}^{V, A}\left(\frac{z-l_{0}}{l_{1}-l_{0}}\right)^{2 d}\right),
$$

with $c_{d} \ll 1$. These coefficients $c_{d}$ parametrize our ignorance of the dynamics responsible for generating the condensates. They could come from several interlaced sources in 5D: unknown string dynamics coupling differently to axial and vector fields, back-reaction of the metric, bulk fields frozen at energies below $l_{0}$, higher order corrections of the YM action,... Building a model for these modifications is out of the scope of this paper, which looks for simplicity (in order to catch the origin of the behavior of 5D models) and aims to interpolate with the known large- $N_{c}$ QCD behavior, rather than to solve QCD.

At linear order in the $c_{d}$, the modification in the two point function is given by ${ }^{11}$

$$
\Pi_{V, A}(Q) \simeq \Pi_{V, A}^{0}(Q)+\sum_{d=2} \frac{\left\langle\mathcal{O}_{2 d}\right\rangle_{V, A}}{Q^{2 d}}
$$

where $\left\langle\mathcal{O}_{2 d}\right\rangle_{V, A}=-c_{d}^{V, A} \frac{d^{2} \sqrt{\pi}}{l_{1}^{2 d}} \frac{\Gamma(d)^{3}}{\Gamma\left(d+\frac{1}{2}\right)}$. The first term $\Pi_{V, A}^{0}(Q)$ was already given in (7.10) and contains the zero-order condensate and the exponential fall-off (7.14). The scale of the condensate is tied with the IR scale, $l_{1}$, and will be responsible for the breaking of the generalized Weinberg sum rules (7.14, D.20) down to the usual two Weinberg Sum rules.

Note at this point that our aim is not to adjust the coefficients $c_{d}^{V, A}$ to the OPE one by one, but to extract information on these high energy parameters by looking at their subsequent effects on the mismatch with $N_{c}=3$, resonance spectrum, $\Gamma\left(a_{1} \longrightarrow \pi \gamma\right)$, axial form factor... and in turn extract some of the condensates.

\section{Conclusions}

We have presented a model of GBs and an infinite number of spin-1 resonances. It is based on a setup with $\mathrm{SU}\left(N_{f}\right) \times \mathrm{SU}\left(N_{f}\right)$ YM fields living in five dimensions (5D), with

\footnotetext{
${ }^{11}$ We thank Arcadi Santamaria for invaluable help on this point.
} 
appropriate boundary conditions. This provides a description of four-dimensional (4D) mesons: massless pions and vector and axial resonances. The 5D gauge symmetry and conformality of the geometry along the extra dimension provide the correct symmetries to link with QCD, interpolating between Chiral Perturbation Theory and large- $N_{c}$ QCD. The model in its simplest form contains two parameters.

Note that we have treated gravity as non-dynamical. Its only effect is to provide a particular metric, which determines the gauge field profiles and the condensates. Wether such a metric can be obtained by solving a dynamical higher-dimensional setup or it is just mimicking effects of quark confinement is out of the scope of this paper.

The simplicity and computability of the two-parameter model is manifest when one compares the inputs (the above-mentioned 5D symmetries) with the outputs. In particular, we have explored all the energy ranges where the model is computable: (i) at low energies, we have described the pion interactions in terms the low energy constants $\left(L_{i}\right.$ 's) of the chiral lagrangian, (ii) we have described resonance interactions at intermediate energies and (iii) we have obtained the two-point functions and form factors as compact expressions involving the $5 \mathrm{D}$ propagator; these can be computed in the high-energy limit, as well as the low-energy one. Finally, (iv) we have described the relation between 4D and 5D descriptions. We now summarize the results point by point.

(i) Low energies. We have used field redefinitions in order to explicitly exhibit the low-energy properties. This applies in particular to the symmetries and interactions of the pions. We have shown that, even in such a constrained setup, one can obtain low energy constants in agreement with experiment. Regarding the Green's functions, we have obtained compact expressions involving the 5D propagator. These match the low-energy chiral expansion, in which non-local order parameters appear.

(ii) Resonances. From a 4D point of view, the model describes an infinite number of narrow spin-1 resonances. A crucial point is that these descend from a 5D Yang-Mills lagrangian. Therefore, their couplings are given by overlap integrals of their wave-functions in the fifth dimension. This means that all resonance couplings (a) are uniquely given as a function of the gauge coupling once the metric is known, and (b) satisfy sum rules reflecting the $5 \mathrm{D}$ origin of the model. We find that dominance by th lightest vector meson $(\rho)$ is automatic.

(iii) High energies. We were also able to compare the behavior of the Green's functions at large euclidean momenta with the operator product expansion of QCD. In its simpler version, the model lacks the ingredient of non-vanishing local order parameters of QCD. This is remedied without introducing new degrees of freedom, as described in Section 7.4.

We have shown that the model yields an infinite number of Weinberg sum rules (and only two when the condensates are introduced), that extended vector meson dominance was automatic, and that the vector form factor followed an unsubtracted dispersion relation.

(iv) $4 D$ and $5 D$ point of view. In the $5 \mathrm{D}$ model, moments of the left-right two point correlators are well-defined. The Weinberg sum rules are obeyed, and generalizations thereof. The 5D result is in itself all we need, but we have also shown how to interpret it in the Kaluza-Klein picture. We have discussed the prescription provided by the extradimensional setup for handling the divergent sums over the infinite number of resonances, 
which has always been an issue for 4D models. Divergences cancel between contributions from the tower of vector resonances and its axial counterpart, because both descend from $5 \mathrm{D}$ fields living in the same extra-dimension.

\section{Acknowledgments}

We benefited from lengthy discussion with Toni Pich, Jorge Portolés, Ximo Prades, Nuria Rius, Arcadi Santamaria, Juanjo Sanz-Cillero and Yael Shadmi. We also thank them for a critical reading of a draft of this paper. VS thanks Ami Katz for sharing his results (during the workshop The weak scale, the Planck scale and what's in-between organized by Yael Shadmi and supported by The Institute of Theoretical Physics, Technion), as well as Alex Pomarol. JH is grateful to Marc Knecht and Jan Stern for advice and for their opinion about the draft, and is very happy to thank Bruno Julia-Díaz for his continuous encouragement to work on the form factor. JH is supported by the European network EURIDICE, contract HPRN-CT-2002-00311 and by the Generalitat Valenciana, contract GV05/015.

\section{A. Field redefinitions}

In this Appendix we show how to go from the model in terms of the original 5D fields in (3.4), $L_{M}, R_{M}$ to the $4 \mathrm{D}$ fields in (3.7), $U, A_{\mu}^{(n)}, V_{\mu}^{(n)}, \ell_{\mu}$ and $r_{\mu}$. These last fields separate clearly between sources and fields, and show that indeed the pion enjoys the correct transformation properties.

The main property of the Wilson line is

$$
\xi_{R}(x, z) \longmapsto R(x, z) \xi_{R}(x, z) h(x)^{\dagger} .
$$

Its covariant derivatives are defined as

$$
\begin{aligned}
D_{\mu} \xi_{R}(x, z) & \equiv \partial_{\mu} \xi_{R}(x, z)-\mathrm{i} R_{\mu}(x, z) \xi_{R}(x, z)+\mathrm{i} \xi_{R}(x, z) h_{\mu}(x), \\
D_{5} \xi_{R}(x, z) & \equiv \partial_{5} \xi_{R}(x, z)-\mathrm{i} R_{5}(x, z) \xi_{R}(x, z) .
\end{aligned}
$$

Similar definitions apply to the case of the $L_{M}$ gauge field as well. $h_{\mu}$ is the common value of $L_{\mu}$ and $R_{\mu}$ at $l_{1}$.

Of particular interest is the Wilson line $\xi$ extending from one boundary to the other $\xi_{R}\left(x, l_{0}\right)$. Other $4 \mathrm{D}$ fields that are useful for computations can be constructed. The first is denoted by $u_{\mu}$, and contains the non-linear derivative of the pion field. This is a more fundamental object than $U$ since GB interactions can only appear with powers of momenta

$$
\begin{aligned}
u_{\mu}(x) & \equiv \mathrm{i}\left\{\xi_{R}^{\dagger}\left(x, l_{0}\right) D_{\mu} \xi_{R}\left(x, l_{0}\right)-\xi_{L}^{\dagger}\left(x, l_{0}\right) D_{\mu} \xi_{L}\left(x, l_{0}\right)\right\} \\
& =\mathrm{i}\left\{\xi_{R}^{\dagger}\left(x, l_{0}\right)\left(\partial_{\mu}-\mathrm{i} r_{\mu}\right) \xi_{R}\left(x, l_{0}\right)-\xi_{L}^{\dagger}\left(x, l_{0}\right)\left(\partial_{\mu}-\mathrm{i} \ell_{\mu}\right) \xi_{L}\left(x, l_{0}\right)\right\} .
\end{aligned}
$$

In the above, the terms involving the $h_{\mu}$ connection in the covariant derivative (A.2) cancel out, and we have

$$
u_{\mu} \longmapsto h u_{\mu} h^{\dagger}
$$


Also of use is the orthogonal combination, which transforms as a connection for the 4D transformation $h(x)$

$$
\begin{aligned}
\Gamma_{\mu}(x) & \equiv \frac{1}{2}\left\{\xi_{R}^{\dagger}\left(x, l_{0}\right)\left(\partial_{\mu}-\mathrm{i} r_{\mu}\right) \xi_{R}\left(x, l_{0}\right)+\xi_{L}^{\dagger}\left(x, l_{0}\right)\left(\partial_{\mu}-\mathrm{i} \ell_{\mu}\right) \xi_{L}\left(x, l_{0}\right)\right\} \\
\Gamma_{\mu} & \longmapsto h \Gamma_{\mu} h^{\dagger}+\mathrm{i} h \partial_{\mu} h^{\dagger}
\end{aligned}
$$

The result $\hat{V}_{5}=\hat{A}_{5}=0$ in equation 3.12 may seem surprising. Since the fifth component of the gauge fields contain the GB fields, we cannot get rid of them in the bulk without conflicting with the BCs [59, 20]: the pion is a physical field and cannot be eliminated. There is no contradiction here, since we have defined new fields $\hat{V}_{M}, \hat{A}_{M}$, whose BCs still have to be derived from those imposed on the original fields $R_{M}, L_{M}$. In particular, we find that the pion field has not disappeared, since we have the relation (3.14), and hence the redefinition (3.15) ${ }^{12}$. Note also that the expression of $\hat{V}_{\mu}$ on the UV brane involves the sources, through (3.13) ${ }^{13}$ where $\hat{V}_{\mu}\left(x, l_{0}\right)=\mathrm{i} \Gamma_{\mu}(x)$.

At this stage, we have used field redefinitions eliminating the $5 \mathrm{D}$ fields $R_{M}, L_{M}$ in favor of the $z$-dependent fields $V_{\mu}, A_{\mu}$ and the $4 \mathrm{D}$ field $u_{\mu}$. In particular, no fifth component of any vector field will occur in the lagrangian. To see how the rewritings lead to the lagrangian $\mathcal{O}\left(p^{2}\right)(4.3)$, we focus on the term that involves indices 5 in the YM action (2.3). We shall need the identities

$$
\begin{aligned}
& L_{5 \mu}=\xi_{L}\left(\partial_{5} V_{\mu}+\partial_{5} A_{\mu}-\frac{1}{2}\left(\partial_{5} \alpha\right) u_{\mu}\right) \xi_{L}^{\dagger}, \\
& R_{5 \mu}=\xi_{R}\left(\partial_{5} V_{\mu}-\partial_{5} A_{\mu}+\frac{1}{2}\left(\partial_{5} \alpha\right) u_{\mu}\right) \xi_{R}^{\dagger},
\end{aligned}
$$

which follow from using the redefinitions (3.13, 3.15). $\alpha(z)$ is a solution of a massless EOM (4.4). For flat space and RS1 respectively, the solution of the EOM for $\alpha$ derived in (4.4), and satisfying the BCs (3.20 3.21) is, respectively

$$
\begin{gathered}
\left.\alpha(z)\right|_{\mathrm{FS}}=1-\frac{z}{l_{1}} \\
\left.\alpha(z)\right|_{\mathrm{RS} 1}=1-\frac{z^{2}-l_{0}^{2}}{l_{1}^{2}-l_{0}^{2}} .
\end{gathered}
$$

The $\mathcal{O}\left(p^{4}\right)$ lagrangian is obtained from the terms with two 4D indices in the YM action

$$
\left\langle R_{\mu \nu} R_{\rho \sigma}+L_{\mu \nu} L_{\rho \sigma}\right\rangle=\frac{1}{2}\left\langle F_{+\mu \nu} F_{+\rho \sigma}+F_{-\mu \nu} F_{-\rho \sigma}\right\rangle,
$$

where the new field strengths $F_{ \pm \mu \nu}$ are defined as

$$
F_{ \pm \mu \nu}(x, z) \equiv \xi_{L}^{\dagger} L_{\mu \nu} \xi_{L} \pm \xi_{R}^{\dagger} R_{\mu \nu} \xi_{R}
$$

We shall also need the definitions of the field strengths of the sources

$$
f_{ \pm \mu \nu}(x) \equiv \xi_{L}^{\dagger}\left(x, l_{0}\right) \ell_{\mu \nu} \xi_{L}\left(x, l_{0}\right) \pm \xi_{R}^{\dagger}\left(x, l_{0}\right) r_{\mu \nu} \xi_{R}\left(x, l_{0}\right) .
$$

\footnotetext{
${ }^{12}$ Comparing with equation (48) of $[11]$, we see that their constant $c$ has turned into a function depending only on the additional coordinate $z$.

${ }^{13}$ The equation corresponds to the one used to obtain (43) in [11].
} 
One can show that $F_{+\mu \nu}$ contains the field-strength of the vector field, the field-strength of the vector source $f_{+\mu \nu}$ and terms involving GBs, as follows

$$
\begin{aligned}
F_{+\mu \nu} & =2\left(\nabla_{\mu} V_{\nu}-\nabla_{\nu} V_{\mu}-\mathrm{i}\left[V_{\mu}, V_{\nu}\right]-\mathrm{i}\left[A_{\mu}, A_{\nu}\right]\right) \\
& +\mathrm{i} \alpha\left(\left[u_{\mu}, A_{\nu}\right]+\left[A_{\mu}, u_{\nu}\right]\right)+f_{+\mu \nu}+\mathrm{i} \frac{1-\alpha^{2}}{2}\left[u_{\mu}, u_{\nu}\right] .
\end{aligned}
$$

We also obtain the following identity for $F_{-\mu \nu}$, in terms of the field strength of the axial field, of the field strength of the axial source $f_{-\mu \nu}$, and additional terms involving GBs

$$
\begin{aligned}
F_{-\mu \nu} & =2\left(\nabla_{\mu} A_{\nu}-\nabla_{\nu} A_{\mu}-\mathrm{i}\left[V_{\mu}, A_{\nu}\right]-\mathrm{i}\left[A_{\mu}, V_{\nu}\right]\right) \\
& +\mathrm{i} \alpha\left(\left[u_{\mu}, V_{\nu}\right]+\left[V_{\mu}, u_{\nu}\right]\right)+\alpha f_{-\mu \nu} .
\end{aligned}
$$

In the above, we have used the standard definition for the covariant derivative of fields transforming under the adjoint of $h(x), \nabla_{\mu} \cdot=\partial_{\mu} \cdot+\left[\Gamma_{\mu}, \cdot\right]$ [10], with $\Gamma_{\mu}$ as defined in (A.7). Assuming now $N_{f}=3$, we get

$$
\begin{aligned}
& -\frac{1}{4 g_{5}^{2}} \int_{l_{0}}^{l_{1}} \mathrm{~d} z \sqrt{g} g^{\mu \rho} g^{\nu \sigma}\left\langle R_{\mu \nu}(z) R_{\rho \sigma}(z)+L_{\mu \nu}(z) L_{\rho \sigma}(z)\right\rangle \\
= & L_{1}\left\langle u_{\mu} u^{\mu}\right\rangle^{2}+L_{2}\left\langle u_{\mu} u_{\nu}\right\rangle\left\langle u^{\mu} u^{\nu}\right\rangle+L_{3}\left\langle u_{\mu} u^{\mu} u_{\nu} u^{\nu}\right\rangle \\
- & \mathrm{i} L_{9}\left\langle f_{+\mu \nu} u^{\mu} u^{\nu}\right\rangle+\frac{1}{4} L_{10}\left\langle f_{+\mu \nu} f_{+}^{\mu \nu}-f_{-\mu \nu} f_{-}^{\mu \nu}\right\rangle \\
+ & \frac{1}{2} H_{1}\left\langle f_{+\mu \nu} f_{+}^{\mu \nu}+f_{-\mu \nu} f_{-}^{\mu \nu}\right\rangle+\text { terms involving resonances. }
\end{aligned}
$$

We have already used the same names as in [5] for the coefficients of the operators. However, in this model, they are not unknown, but rather predicted in terms of the two free parameters of the model. The expressions for a generic metric are given in (4.9-4.14).

\section{B. Resonance wave-functions}

The EOM for the resonances (5.2) becomes, when using the conformally flat coordinates (2.2)

$$
-\frac{1}{w(z)} \partial_{z}\left(w(z) \partial_{z}\right) \varphi_{n}^{X}=M_{X_{n}}^{2} \varphi_{n}^{X},
$$

where $X=V, A$. Vector and axial cases only differ by the BCs. For the vector case,

$$
\begin{array}{r}
\left.\varphi_{n}^{V}(z)\right|_{z=l_{0}}=0, \\
\left.\partial_{z} \varphi_{n}^{V}(z)\right|_{z=l_{1}}=0,
\end{array}
$$

and for the axial one $\left.\varphi_{n}^{A}(z)\right|_{z=l_{0}}=\left.\varphi_{n}^{A}(z)\right|_{z=l_{1}}=0$.

We normalize the KK wave-functions $\varphi_{n}$ by imposing on them

$$
\frac{2}{g_{5}^{2}} \int_{l_{0}}^{l_{1}} \mathrm{~d} z \sqrt{g} g^{\mu \nu} g^{\rho \sigma} \varphi_{m}^{V} \varphi_{n}^{V}=\delta_{m n} \eta^{\mu \nu} \eta^{\rho \sigma} .
$$


With this normalization, one obtains the following quadratic terms for the $4 \mathrm{D}$ KK modes

$$
\begin{aligned}
& -\frac{1}{2 g_{5}^{2}} \int_{l_{1}}^{l_{0}} \mathrm{~d} z \sqrt{g} g^{\mu \nu}\left\{g^{\rho \sigma}\left\langle\left(\partial_{\mu} V_{\rho}-\partial_{\rho} V_{\mu}\right)\left(\partial_{\nu} V_{\sigma}-\partial_{\sigma} V_{\nu}\right)\right\rangle+2 g^{55}\left\langle\partial_{5} V_{\mu} \partial_{5} V_{\nu}\right\rangle\right\} \\
= & -\frac{1}{4} \eta^{\mu \nu} \sum_{n=1}^{\infty}\left(\eta^{\rho \sigma}\left\langle\left(\partial_{\mu} V_{\rho}^{(n)}-\partial_{\rho} V_{\mu}^{(n)}\right)\left(\partial_{\nu} V_{\sigma}^{(n)}-\partial_{\sigma} V_{\nu}^{(n)}\right)\right\rangle-2 M_{V_{n}}^{2}\left\langle V_{\mu}^{(n)} V_{\nu}^{(n)}\right\rangle\right)(\mathrm{B} .5
\end{aligned}
$$

The second term in the right-hand side of the above is the sum of the mass terms for the KK modes.

For the flat case, i.e. $w(z)=1$, the solutions to the EOMs for resonances (B.1) with BCs appropriate to the vector and axial case are harmonic functions

$$
\varphi_{n}^{V, A}(z)=\frac{1}{g_{4}} \sin \left(M_{V_{n}, A_{n}} z\right)
$$

where we have used the normalization (B.4). The masses are given in Table 3.

For the warp factor $w(z)=l_{0} / z$, corresponding to the RS1 case, the general solution to the EOMs for resonances is written in terms of Bessel functions

$$
\varphi_{n}^{X}(z)=\frac{z}{g_{4} N_{n}^{X}}\left(J_{1}\left(M_{X_{n}} z\right)+\alpha_{n}^{X} Y_{1}\left(M_{X_{n}} z\right)\right)
$$

with the dimensionful normalization factor

$$
\left(N_{n}^{X}\right)^{2}=2 \int_{l_{0}}^{l_{1}} \mathrm{~d} z z\left(J_{1}\left(M_{X_{n}} z\right)+\alpha_{n}^{X} Y_{1}\left(M_{X_{n}} z\right)\right)^{2} .
$$

The relevant BCs for the vector field (B.2 $\mathrm{B} .3)$ impose

$$
\begin{aligned}
\alpha_{n}^{V} & =-\frac{J_{1}\left(M_{V_{n}} l_{0}\right)}{Y_{1}\left(M_{V_{n}} l_{0}\right)}, \\
\frac{J_{0}\left(M_{V_{n}} l_{1}\right)}{Y_{0}\left(M_{V_{n}} l_{1}\right)} & =-\frac{J_{1}\left(M_{V_{n}} l_{0}\right)}{Y_{1}\left(M_{V_{n}} l_{0}\right)},
\end{aligned}
$$

while for the axial case, we find

$$
\begin{aligned}
\alpha_{n}^{A} & =-\frac{J_{1}\left(M_{A_{n}} l_{0}\right)}{Y_{1}\left(M_{V_{n}} l_{0}\right)}, \\
\frac{J_{1}\left(M_{A_{n}} l_{1}\right)}{Y_{1}\left(M_{A_{n}} l_{1}\right)} & =\frac{J_{1}\left(M_{A_{n}} l_{0}\right)}{Y_{1}\left(M_{A_{n}} l_{0}\right)} .
\end{aligned}
$$

This allows us to determine numerically both the masses $M_{V_{n}}, M_{A_{n}}$ and the corresponding coefficients $\alpha_{n}^{V, A}$ in the linear combinations. For resonances sufficiently light compared to the regulator $1 / l_{0}$, or assuming that the regulator is removed, one can obtain approximate expressions since the coefficient fixing the linear combination is small

$$
\alpha_{n}^{V, A} \simeq \frac{\pi}{4}\left(M_{V_{n}, A_{n}} l_{0}\right)^{2} \ll 1
$$

Plugging this into the expression for the wave-functions $(\sqrt{B .7})$, one finds that the masses are approximately determined by the zeroes of the Bessel functions

$$
\begin{aligned}
& J_{0}\left(M_{V_{n}} l_{1}\right) \simeq 0, \\
& J_{1}\left(M_{A_{n}} l_{1}\right) \simeq 0 .
\end{aligned}
$$


This can be solved for numerically, or alternatively, one can obtain the approximate expressions given in Table 3 .

\section{Resonance couplings}

The terms involving resonances in (A.17) can be written as in 51 generalized with an index $n$

$$
\begin{aligned}
& -\frac{1}{4 g_{5}^{2}} \int \mathrm{d} z \sqrt{g} g^{\mu \nu} g^{\rho \sigma}\left\langle R_{\mu \rho}(z) R_{\nu \sigma}(z)+L_{\mu \rho}(z) L_{\nu \sigma}(z)\right\rangle \\
\supset & -\frac{1}{2 \sqrt{2}} \sum_{n=1}^{\infty}\left\{f_{V_{n}}\left\langle\left(\nabla_{\mu} V_{\nu}^{(n)}-\nabla_{\nu} V_{\mu}^{(n)}\right) f_{+}^{\mu \nu}\right\rangle+\mathrm{i} g_{V_{n}}\left\langle\left(\nabla_{\mu} V_{\nu}^{(n)}-\nabla_{\nu} V_{\mu}^{(n)}\right)\left[u^{\mu}, u^{\nu}\right]\right\rangle\right. \\
+ & \sum_{n=1}^{\infty}\left\{-\frac{1}{2 \sqrt{2}} f_{A_{n}}\left\langle\left(\nabla_{\mu} A_{\nu}^{(n)}-\nabla_{\nu} A_{\mu}^{(n)}\right) f_{-}^{\mu \nu}\right\rangle+\mathrm{i} \alpha_{A_{n}}\left\langle\left[A_{\mu}^{(n)}, u_{\nu}\right] f_{+}^{\mu \nu}\right\rangle\right\}+\ldots
\end{aligned}
$$

where we have only singled out some of the interaction terms. The expressions for the resonance couplings to sources $f_{V_{n}}, f_{A_{n}}$ are

$$
\begin{aligned}
& \eta^{\mu \nu} \eta^{\rho \sigma} f_{V_{n}}=\frac{\sqrt{2}}{g_{5}^{2}} \int_{l_{0}}^{l_{1}} \mathrm{~d} z \sqrt{g} g^{\mu \nu} g^{\rho \sigma} \varphi_{n}^{V} \\
& \eta^{\mu \nu} \eta^{\rho \sigma} f_{A_{n}}=\frac{\sqrt{2}}{g_{5}^{2}} \int_{l_{0}}^{l_{1}} \mathrm{~d} z \sqrt{g} g^{\mu \nu} g^{\rho \sigma} \alpha \varphi_{n}^{A} .
\end{aligned}
$$

The coupling of one vector resonance to two pions is given by

$$
\eta^{\mu \nu} \eta^{\rho \sigma} g_{V_{n}}=\frac{\sqrt{2}}{2 g_{5}^{2}} \int_{l_{0}}^{l_{1}} \mathrm{~d} z \sqrt{g} g^{\mu \nu} g^{\rho \sigma}\left(1-\alpha^{2}\right) \varphi_{n}^{V} .
$$

For the decay constants, i.e. terms that contain a quadratic coupling between a source and a field, one can obtain expressions involving derivatives at the UV boundary, rather than bulk integrals. This is done using integration by parts as well as the EOMs and BCs for both $\alpha$ and $\varphi_{n}$. The expressions for the resonance decay constants $f_{V_{n}, A_{n}}$ may then be recast in the form

$$
\begin{gathered}
\eta^{\mu \nu} f_{V_{n}} M_{V_{n}}^{2}=-\left.\frac{\sqrt{2}}{g_{5}^{2}} \sqrt{g} g^{\mu \nu} g^{55} \partial_{5} \varphi_{n}^{V}\right|_{l_{0}} \\
\eta^{\mu \nu} f_{A_{n}} M_{A_{n}}^{2}=-\left.\frac{\sqrt{2}}{g_{5}^{2}} \sqrt{g} g^{\mu \nu} g^{55} \partial_{5} \varphi_{n}^{A}\right|_{l_{0}} .
\end{gathered}
$$

These expressions (C.5 C.6) have a more holographic twist than (C.2 C.3), since they give the couplings of $\mathrm{KK}$ modes as boundary values of derivatives with respect to the fifth coordinate of the wave-functions, rather than in terms of bulk integrals.

Note as well that a similar equivalence can be proved for the pion decay constant, using again integration by parts to rewrite (4.6) as

$$
\eta^{\mu \nu} f^{2}=\left.\frac{1}{g_{5}^{2}} \sqrt{g} g^{\mu \nu} g^{55} \partial_{5} \alpha\right|_{l_{0}} .
$$




\section{5D regularization of infinite sums}

The 5D origin of the model means that the different resonance couplings are given by overlap integrals of wave-functions $\varphi_{n}(z)$, which obey completeness relations ${ }^{14}$

$$
\frac{2}{g_{5}^{2}} \sqrt{g} g^{\mu \nu} g^{\rho \sigma} \sum_{n=1}^{\infty} \varphi_{n}^{V}(z) \varphi_{n}^{V}\left(z^{\prime}\right)=\eta^{\mu \nu} \eta^{\rho \sigma} \delta\left(z-z^{\prime}\right) .
$$

Using this remark, we were able to derive the sum rules of Section 6.1, which stipulate that sums of products of resonance couplings are related to GB interactions. The sums 6.1-6.4 were convergent, so no problems were encountered there. We now focus on the sums $\sum_{n} f_{V_{n}}^{2}$ and $\sum_{n} f_{A_{n}}^{2}$, which are logarithmically divergent for the case of the RS1 metric in the limit $l_{0} \longrightarrow 0$, see Table 5

In principle, one has to relate the two regulators for the infinite sums in a way that preserves chiral symmetry, as advocated in [16]. The usual 4D procedure involves cutting the infinite sums over $n$, but chopping the KK tower would be inconsistent with $5 \mathrm{D}$ gauge invariance. However, we can rewrite the sums as integrals over the fifth coordinate, obtaining

$$
\sum_{n=1}^{\infty} f_{A_{n}}^{2}=\frac{1}{g_{5}^{2}} \int_{l_{0}}^{l_{1}} \mathrm{~d} z w(z) \alpha^{2}(z),
$$

for the axial case and (7.24) for the vector one. The extra-dimensional setup yields a clear prescription which preserves the $\mathrm{SU}\left(N_{f}\right) \times \mathrm{SU}\left(N_{f}\right)$ symmetry. It involves combining $\sum_{n} f_{V_{n}}^{2}(\overline{7.24})$ and $\sum_{n} f_{A_{n}}^{2}(\overline{\mathrm{D} .2})$ as

$$
\sum_{n=1}^{\infty} f_{V_{n}}^{2}-\sum_{n=1}^{\infty} f_{A_{n}}^{2}=\frac{1}{g_{5}^{2}} \int_{l_{0}}^{l_{1}} \mathrm{~d} z w(z)\left(1-\alpha^{2}(z)\right),
$$

and sending the regulator $1 / l_{0}$ to infinity afterwards. Since the $\mathrm{BC}(3.20)$ imposes $\alpha\left(l_{0}\right)=$ 1 , the result is finite

$$
\sum_{n=1}^{\infty}\left(f_{V_{n}}^{2}-f_{A_{n}}^{2}\right)=-4 L_{10}
$$

This expression generalizes the one known for the case of a finite number of resonance. The result (D.4) is also valid for any other metric where the integral in the right-hand side of (D.3) converges: for flat space in particular, each one of the two sums is separately convergent.

For RS1, the method described above is the appropriate way to handle the divergence, since vector and axial fields live in the same extra dimension, and both come from linear combinations of the original $L_{\mu}$ and $R_{\mu}$ fields. Rewriting the sums over $n$ as integrals

\footnotetext{
${ }^{14}$ This relation is valid when the distribution is applied to a function satisfying the same BCs as $\varphi_{n}^{V}$. For functions that do not satisfy these BCs, one obtains additional boundary terms, which would be missed by blindly applying (D.1).
} 
over the bulk with the regulator $1 / l_{0}$ present takes this into account, while preserving the $\mathrm{SU}\left(N_{f}\right) \times \mathrm{SU}\left(N_{f}\right)$ symmetry. Removing the regulator then yields the result (D.4).

The prescription is clearly dictated by the fact that the model is defined in an extradimensional setup. As a matter of fact, working in 5D, none of these subtleties would be necessary. The result is transparent and no ordering in taking the limits is necessary since the only scales are the momentum $q$ and the position of the IR brane $l_{1}$. No intermediate KK description is needed: in fact, the description in terms of resonances hides the local structure of the fifth dimension. In order to preserve this structure, one can still use the $4 \mathrm{D}$ representation in terms of sums, albeit with a regularization appropriate to 5D. One example is the so-called $K K$ regularization of [60], which we consider in Section D.1.

We are going to see how one obtains the two Weinberg sum rules (WSRs) and their generalizations by considering moments of the $\Pi_{L R}$ correlator

$$
\text { for } k \geqslant 1, \quad \frac{1}{\pi} \int_{0}^{\infty} \mathrm{d} s s^{k-1} \operatorname{Im} \Pi_{L R}(s)=\sum_{n=1}^{\infty} f_{V_{n}}^{2} M_{V_{n}}^{2 k}-\sum_{n=1}^{\infty} f_{A_{n}}^{2} M_{A_{n}}^{2 k}-f^{2} \delta^{k 1}
$$

Note that the case of $L_{10}$ studied above corresponds to $k=0$ with the pion pole removed. The WSRs are closely related to the values of the OPE coefficients in $\Pi_{L R}$ (7.20): we will find that the sums (D.5) vanish, as expected from (7.20).

\section{D.1 KK regularization}

Limiting ourselves to flat space, we assume that the (electroweak) matter content is spread with a Gaussian distribution, rather than being localized as a delta function on the UV brane. The spread of the Gaussian in the fifth coordinate would be proportional to a length $\sqrt{\tau}$. The (electroweak) decay constants are then expected to be modified as

$$
f_{V_{n}}^{2} \rightarrow f_{V_{n}}^{2} \mathrm{e}^{-\tau M_{V_{n}}^{2}}
$$

and similarly for the axial case. The regulator $\tau$ should be sent to zero at the end of the computations.

We now directly define the separate regulated sums representing the vector and axial contributions to the $k$-th moment of (D.5)

$$
\begin{aligned}
& V^{(k)}(\tau) \equiv \sum_{n=1}^{\infty} f_{V_{n}}^{2} \mathrm{e}^{-\tau M_{V_{n}}^{2} M_{V_{n}}^{2 k}} \\
& A^{(k)}(\tau) \equiv \sum_{n=1}^{\infty} f_{A_{n}}^{2} \mathrm{e}^{-\tau M_{A_{n}}^{2}} M_{A_{n}}^{2 k}
\end{aligned}
$$

This regulation makes both sums finite for any $k$ : the powers of masses are compensated by the exponential suppression for any $\tau>0$. We next introduce the variable

$$
q \equiv \mathrm{e}^{-\tau\left(\frac{\pi}{l_{1}}\right)^{2}}
$$


which tends to one as the regulator $\tau$ is sent to zero. We rewrite the sums (D.7 D.8) in terms of elliptic theta functions as

$$
\begin{aligned}
V^{(k)}(\tau) & =\frac{1}{\left(g_{4} l_{1}\right)^{2}}\left(\frac{\pi}{l_{1}}\right)^{2(k-1)}\left[q \frac{\partial}{\partial q}\right]^{k-1} \vartheta_{2}(0, q) \\
A^{(k)}(\tau) & =\frac{1}{\left(g_{4} l_{1}\right)^{2}}\left(\frac{\pi}{l_{1}}\right)^{2(k-1)}\left[q \frac{\partial}{\partial q}\right]^{k-1}\left\{\vartheta_{3}(0, q)-1\right\}
\end{aligned}
$$

The separate sums have been regulated, and only diverge if $q$ is set to 1 . We can however first combine the well-defined vector and axial sum in order to examine the moments of $\Pi_{L R}$ introduced in (D.5). We use

$$
\vartheta_{3}(0, q)-\vartheta_{2}(0, q)=\vartheta_{4}\left(0, q^{1 / 4}\right)
$$

and the fact that $\vartheta_{4}(0, q)$ and all its derivatives vanish as $q \longrightarrow 1$

$$
\text { for } k \geqslant 0 \quad \frac{\partial^{k}}{\partial q^{k}} \vartheta_{4}(0, q) \underset{q \rightarrow 1}{\longrightarrow} 0 \text {. }
$$

With all these rewritings, we obtain that the finite expressions combine to give the expected results in the limit where the regulator is removed

$$
\begin{aligned}
& V^{(1)}(\tau)-A^{(1)}(\tau) \underset{\tau \rightarrow 0}{\longrightarrow} f^{2}, \\
& \text { for } k \geqslant 2, \quad V^{(k)}(\tau)-A^{(k)}(\tau) \underset{\tau \rightarrow 0}{\longrightarrow} 0 .
\end{aligned}
$$

In other words, we reobtain the infinite number of generalized Weinberg sum rules. The $5 \mathrm{D}$ regularization has automatically modified both the vector and axial contributions to yield this result: regulating in $x^{5}$-position space affects both $L_{\mu}$ and $R_{\mu}$ gauge fields, and hence $V_{\mu}$ and $A_{\mu}$ in a definite way, so as to preserve the $\mathrm{SU}\left(N_{f}\right) \times \mathrm{SU}\left(N_{f}\right)$ symmetry.

\section{D.2 Generalized Weinberg sum rules}

Having seen that the axial and vector sums separately diverged, but that the difference could yield a meaningful result, we now work with a generic metric. If we do not insist on regulating the delta functions, we can use the completeness relation (D.1) to rewrite the sums in (D.5) as expressions in $x^{5}$ position-space. The divergences will again cancel between vector and axial contributions. Using the expressions for the resonance couplings C.2 C.6), we find the following expressions for the infinite sums corresponding to the first WSR

$$
\begin{aligned}
\sum_{n=1}^{\infty} f_{V_{n}}^{2} M_{V_{n}}^{2} & =\left.\frac{1}{g_{5}^{2}}\left\{w \delta\left(z-l_{0}\right)+\partial_{z} w\right\}\right|_{z=l_{0}}, \\
\sum_{n=1}^{\infty} f_{A_{n}}^{2} M_{A_{n}}^{2} & =\left.\frac{1}{g_{5}^{2}}\left\{w \delta\left(z-l_{0}\right)+\partial_{z} w+w \partial_{z} \alpha\right\}\right|_{z=l_{0}}
\end{aligned}
$$


These expressions involve boundary terms on the UV brane. The final result is the expected one for the first WSR

$$
\sum_{n=1}^{\infty} f_{V_{n}}^{2} M_{V_{n}}^{2}-\sum_{n=1}^{\infty} f_{A_{n}}^{2} M_{A_{n}}^{2}=f^{2}
$$

For moments with $k \geqslant 2$ in (D.5), one only has to use expressions for the resonance couplings C.5 C.6) together with the completeness relations. The expressions for the vector contribution

$$
\text { for } k \geqslant 2, \quad \sum_{n=1}^{\infty} f_{V_{n}}^{2} M_{V_{n}}^{2 k}=-\left.\frac{1}{g_{5}^{2}}\left\{w \partial_{z}\left(-\frac{1}{w} \partial_{z} w \partial_{z}\right)^{k-2} \partial_{z} \delta\left(z-l_{0}\right)\right\}\right|_{z=l_{0}},
$$

though highly divergent, is strictly equal to that of the axial one. We thus find that the model satisfies an infinite number of generalized WSRs, i.e.

$$
\text { for } k \geqslant 2, \quad \sum_{n=1}^{\infty} f_{V_{n}}^{2} M_{V_{n}}^{2 k}-\sum_{n=1}^{\infty} f_{A_{n}}^{2} M_{A_{n}}^{2 k}=0 .
$$

The 5D expressions used for these infinite sums involve terms localized in the extra dimension, delta functions signaling that our world is living in $l_{0}$. Those divergent contributions cancel between the vector and axial currents. The fact that vector and axial resonances descend from 5D fields living in the same extra-dimension means that the result respects chiral symmetry.

A last comment is in order: a delta function distribution in any $5 \mathrm{D}$ theory must be taken as a regularized delta function. This would give a finite value at intermediate stages for both axial and vector sums, as exemplified in the KK-regularization used in Section D.1.

\section{References}

[1] G. 't Hooft, A planar diagram theory for strong interactions, Nucl. Phys. B72 (1974) 461.

[2] E. Witten, Baryons in the 1/N expansion, Nucl. Phys. B160 (1979) 57.

[3] S. Weinberg, Phenomenological lagrangians, Physica A96 (1979) 327.

[4] J. Gasser and H. Leutwyler, Chiral Perturbation Theory to one loop, Ann. Phys. 158 (1984) 142.

[5] J. Gasser and H. Leutwyler, Chiral Perturbation Theory: Expansions in the mass of the strange quark, Nucl. Phys. B250 (1985) 465.

[6] M. Bando, T. Kugo, S. Uehara, K. Yamawaki, and T. Yanagida, Is the $\rho$ meson a dynamical gauge boson of hidden local symmetry?, Phys. Rev. Lett. 54 (1985) 1215.

[7] U.-G. Meißner, Low-energy hadron physics from effective chiral lagrangians with vector mesons, Phys. Rept. 161 (1988) 213.

[8] M. Bando, T. Fujiwara, and K. Yamawaki, Generalized hidden local symmetry and the $A_{1}$ meson, Prog. Theor. Phys. 79 (1988) 1140. 
[9] J. Bijnens, C. Bruno, and E. de Rafael, Nambu-Jona-Lasinio like models and the low-energy effective action of QCD, Nucl. Phys. B390 (1993) 501, hep-ph/9206236.

[10] G. Ecker, J. Gasser, A. Pich, and E. de Rafael, The role of resonances in Chiral Perturbation Theory, Nucl. Phys. B321 (1989) 311.

[11] G. Ecker, J. Gasser, H. Leutwyler, A. Pich, and E. de Rafael, Chiral lagrangians for massive spin-1 fields, Phys. Lett. B223 (1989) 425.

[12] S. Peris, M. Perrottet, and E. de Rafael, Matching long and short distances in large- $N_{c} Q C D$, JHEP 05 (1998) 011, hep-ph/9805442.

[13] M. A. Shifman, Quark-hadron duality, hep-ph/0009131.

[14] M. Golterman and S. Peris, Large- $N_{c} Q C D$ meets Regge theory: The example of spin-one two-point functions, JHEP 01 (2001) 028, hep-ph/0101098.

[15] M. Golterman, S. Peris, B. Phily, and E. De Rafael, Testing an approximation to large- $N_{c}$ QCD with a toy model, JHEP 01 (2002) 024, hep-ph/0112042.

[16] M. Golterman and S. Peris, On the use of the operator product expansion to constrain the hadron spectrum, Phys. Rev. D67 (2003) 096001, hep-ph/0207060.

[17] S. S. Afonin, A. A. Andrianov, V. A. Andrianov, and D. Espriu, Matching Regge theory to the OPE, JHEP 04 (2004) 039, hep-ph/0403268.

[18] O. Cata, M. Golterman, and S. Peris, Duality violations and spectral sum rules, hep-ph/0506004.

[19] D. T. Son and M. A. Stephanov, QCD and dimensional deconstruction, Phys. Rev. D69 (2004) 065020, hep-ph/0304182.

[20] T. Sakai and S. Sugimoto, Low energy hadron physics in holographic QCD, Prog. Theor. Phys. 113 (2005) 843, hep-th/0412141].

[21] N. Arkani-Hamed, A. G. Cohen, and H. Georgi, Electroweak symmetry breaking from dimensional deconstruction, Phys. Lett. B513 (2001) 232, hep-ph/0105239].

[22] N. Arkani-Hamed, A. G. Cohen, and H. Georgi, (De)constructing dimensions, Phys. Rev. Lett. 86 (2001) 4757, hep-th/0104005.

[23] J. Hirn, From mooses to $5 D$ and back to large- $N_{c} Q C D$ ?, in Bijnens et al. 61. hep-ph/0502008.

[24] J. M. Maldacena, The large N limit of superconformal field theories and supergravity, Adv. Theor. Math. Phys. 2 (1998) 231, hep-th/9711200].

[25] S. S. Gubser, I. R. Klebanov, and A. M. Polyakov, Gauge theory correlators from non-critical string theory, Phys. Lett. B428 (1998) 105, hep-th/9802109].

[26] E. Witten, Anti-de sitter space and holography, Adv. Theor. Math. Phys. 2 (1998) 253, hep-th/9802150.

[27] N. Arkani-Hamed, M. Porrati, and L. Randall, Holography and phenomenology, JHEP 08 (2001) 017, hep-th/0012148.

[28] R. Barbieri, A. Pomarol, and R. Rattazzi, Weakly coupled Higgsless theories and precision electroweak tests, Phys. Lett. B591 (2004) 141, hep-ph/0310285. 
[29] J. Hirn and J. Stern, The role of spurions in Higgs-less electroweak effective theories, Eur. Phys. J. C34 (2004) 447, hep-ph/0401032.

[30] S. Weinberg, Precise relations between the spectra of vector and axial-vector mesons, Phys. Rev. Lett. 18 (1967) 507.

[31] M. A. Shifman, A. I. Vainshtein, and V. I. Zakharov, QCD and resonance physics. Sum rules, Nucl. Phys. B147 (1979) 385.

[32] M. Knecht and E. de Rafael, Patterns of spontaneous chiral symmetry breaking in the large $N_{c}$ limit of QCD-like theories, Phys. Lett. B424 (1998) 335, hep-ph/9712457.

[33] J. Erlich, E. Katz, D. T. Son, and M. A. Stephanov, QCD and a holographic model of hadrons, hep-ph/0501128.

[34] L. Da Rold and A. Pomarol, Chiral symmetry breaking from five dimensional spaces, hep-ph/0501218.

[35] G. von Gersdorff, L. Pilo, M. Quiros, A. Riotto, and V. Sanz, Supersymmetry from boundary conditions, Nucl. Phys. B712 (2005) 3-19, hep-th/0411133].

[36] J. Hirn and J. Stern, Lepton-number violation and right-handed neutrinos in Higgs-less effective theories, hep-ph/0504277.

[37] G. von Gersdorff, N. Irges, and M. Quiros, Finite mass corrections in orbifold gauge theories, hep-ph/0206029.

[38] L. Randall and R. Sundrum, A large mass hierarchy from a small extra dimension, Phys. Rev. Lett. 83 (1999) 3370, hep-ph/9905221.

[39] H. Leutwyler, On the foundations of Chiral Perturbation Theory, Ann. Phys. 235 (1994) 165, hep-ph/9311274.

[40] M. Knecht, La chromodynamique quantique à basse énergie, in Henrard and Perrottet [62].

[41] S. R. Coleman, J. Wess, and B. Zumino, Structure of phenomenological lagrangians. 1, Phys. Rev. 177 (1969) 2239.

[42] D. J. Gross and R. Jackiw, Effect of anomalies on quasi-renormalizable theories, Phys. Rev. D6 (1972) 477.

[43] C. Grosse-Knetter and R. Kögerler, Unitary gauge, Stückelberg formalism and gauge invariant models for effective lagrangians, Phys. Rev. D48 (1993) 2865, hep-ph/9212268].

[44] I. Rosell, J. J. Sanz-Cillero, and A. Pich, Quantum loops in the resonance chiral theory: The vector form factor, JHEP 08 (2004) 042, hep-ph/0407240.

[45] O. Cata and S. Peris, An example of resonance saturation at one loop, Phys. Rev. D65 (2002) 056014, hep-ph/0107062.

[46] J. J. Sanz-Cillero and A. Pich, Rho meson properties in the chiral theory framework, Eur. Phys. J. C27 (2003) 587, hep-ph/0208199.

[47] J. Bijnens, G. Ecker, and J. Gasser, Chiral perturbation theory, in Maiani et al. [63]. hep-ph/9411232.

[48] G. 't Hooft, A two-dimensional model for mesons, Nucl. Phys. B75 (1974) 461.

[49] A. V. Anisovich, V. V. Anisovich, and A. V. Sarantsev, Systematics of $q \bar{q}$-states in the $\left(n, M^{2}\right)$ and $\left(J, M^{2}\right)$ planes, Phys. Rev. D62 (2000) 051502, hep-ph/0003113. 
[50] Particle Data Group Collaboration, S. Eidelman et al., Review of particle physics, Phys. Lett. B592 (2004) 1. http://pdg.1bl.gov/.

[51] J. Prades, Massive spin 1 field chiral lagrangian from an extended Nambu-Jona-Lasinio model of QCD, Z. Phys. C63 (1994) 491, hep-ph/9302246].

[52] M. Knecht and A. Nyffeler, Resonance estimates of $\mathcal{O}\left(p^{6}\right)$ low-energy constants and $Q C D$ short-distance constraints, Eur. Phys. J. C21 (2001) 659, hep-ph/0106034.

[53] J. J. Sakurai, Currents and mesons. Univ. of Chicago Press, 1969.

[54] G. P. Lepage and S. J. Brodsky, Exclusive processes in quantum chromodynamics: Evolution equations for hadronic wave functions and the form-factors of mesons, Phys. Lett. B87 (1979) 359.

[55] G. P. Lepage and S. J. Brodsky, Exclusive processes in perturbative quantum chromodynamics, Phys. Rev. D22 (1980) 2157.

[56] M. Froissart, Asymptotic behavior and subtractions in the Mandelstam representation, Phys. Rev. 123 (1961) 1053.

[57] L. Randall and R. Sundrum, Out of this world supersymmetry breaking, Nucl. Phys. B557 (1999) 79, hep-th/9810155.

[58] S. R. Beane, Constraining quark-hadron duality at large $N_{c}$, Phys. Rev. D64 (2001) 116010, hep-ph/0106022.

[59] R. Contino, Y. Nomura, and A. Pomarol, Higgs as a holographic pseudo-Goldstone boson, Nucl. Phys. B671 (2003) 148, hep-ph/0306259.

[60] A. Delgado, G. von Gersdorff, P. John, and M. Quiros, One-loop Higgs mass finiteness in supersymmetric Kaluza- Klein theories, Phys. Lett. B517 (2001) 445, hep-ph/0104112.

[61] J. Bijnens, U.-G. Meissner, and A. Wirzba, eds., Mini-proceedings of the EFT04 Workshop on Effective Field Theories in Nuclear Particle and Atomic Physics, Bad Honnef, Germany, December 13-17, 2004, 2005.

[62] P. Henrard and M. Perrottet, eds., La chromodynamique quantique sous toutes ses couleurs, IN2P3, 1996.

[63] L. Maiani, G. Pancheri, and N. Paver, eds., The second DAPHNE physics handbook, INFN, 1995. 